\title{
Deformations of quantum field theories and integrable models
}

\author{
Gandalf Lechner* \\ Faculty of Physics, University of Vienna, \\ Boltzmanngasse 5, A-1090 Vienna, Austria \\ gandalf.lechner@univie.ac.at
}

April 24, 2022

\begin{abstract}
Deformations of quantum field theories which preserve Poincaré covariance and localization in wedges are a novel tool in the analysis and construction of model theories. Here a general scenario for such deformations is discussed, and an infinite class of explicit examples is constructed on the Borchers-Uhlmann algebra underlying Wightman quantum field theory. These deformations exist independently of the space-time dimension, and contain the recently studied warped convolution deformation as a special case. In the special case of two-dimensional Minkowski space, they can be used to deform free field theories to integrable models with non-trivial S-matrix.
\end{abstract}

\section{Introduction}

In the last years, many new quantum field theoretic models have been constructed with nonstandard methods SSch97, SW00, BGL02, Lec03, LR04, Lec05, MSY06, GL07, BS07, BS08, GL08, BLS10, LW10, DT10. Among the different approaches used for constructing these models, a recurring theme is to start with a well-understood model (like a free field theory), and then apply some kind of deformation to change it to a model with non-trivial interaction. As is well known, it is extremely complicated to carry out such a procedure on a non-perturbative level when requiring that it should keep the full covariance, spectral and locality properties of quantum field theory intact. However, interesting manageable examples do exist when the locality requirements are somewhat weakened.

More precisely, there exist many models of quantum fields which are not point-like localized, but rather localized in certain unbounded, wedge-shaped regions (wedges) in Minkowski space [SW00, BGL02, Lec03, LR04, BS07, GL07, BLS10]. These models are still fully Poincaré covariant and comply with Einstein causality inasmuch that observables with spacelike separated wedges commute. Using the algebraic framework of quantum field theory [Haa96, it is also in principle possible BL04 to extract all observables localized in bounded spacetime regions. Moreover, the localization in wedges is sharp enough to consistently compute the two-particle scattering matrix BBS01, and decide if the constructed model exhibits non-trivial interaction.

In view of these facts, wedge-local quantum field theories have many of the characteristic features of fully local quantum field theories, and understanding their structure is an important intermediate step in the rigorous construction of interacting models. It is therefore interesting

\footnotetext{
*Supported by FWF project P22929-N16 "Deformations of Quantum Field Theories".
} 
to note that it is possible to construct wedge-local quantum field theories non-perturbatively, and introduce non-trivial interaction by deformation techniques.

A particular deformation of this kind, based on actions of the translation group, is by now well understood. After its first appearance in the context of deformed free field theories on noncommutative Minkowski space GL07, it was generalized to an operator-algebraic setting in [BS08, where it is known as warped convolution. In the framework of Wightman field theories, this deformation manifests itself as a deformation of the tensor product of the testfunction algebra GL08, and later on, the connection to Rieffel's strict deformation quantization Rie92 was explored [BLS10]. By now, the warped convolution technique has also successfully been applied to the deformation of conformal field theories [DT10] and quantum field theories on curved spacetimes [DLM11.

In this paper we start to explore more general deformations of wedge-local quantum field theories. As a first scenario for such deformations, we focus here on Wightman quantum field theories [SW64, Jos65]. Any Wightman quantum field theory is given by a specific representation of the tensor algebra $\underline{\mathscr{S}}$ over Schwartz' function space $\mathscr{S}\left(\mathbb{R}^{d}\right)$. The deformations studied here are based on linear homeomorphisms $\rho: \mathscr{S} \rightarrow \underline{\mathscr{S}}$ commuting with the natural Poincaré automorphisms $\alpha_{x, \Lambda}$ on $\mathscr{\mathscr { S }}$, for $(x, \Lambda)$ in a subgroup of the Poincaré group which models the geometry of a reference wedge. We then equip $\mathscr{S}$ with a family of new products, namely $f, g \mapsto \rho^{-1}(\rho(f) \otimes \rho(g))$, and Lorentz transforms thereof. Every single of these products provides only a trivial deformation of the tensor product $\otimes$, but their interplay with the local structure of $\mathscr{S}$ gives rise to non-trivial deformations of a net of algebras localized in wedges. If a compatibility condition between $\rho$ and a state $\omega$ on $\mathscr{S}$ is met, one can pass to suitable GNS representations, where all twisted product structures are represented on the same Hilbert space. Here we obtain new quantum field theoretic models, which are wedge-local under further conditions on the deformation map $\rho$ and the state $\omega$.

In Section 2, we explain these deformations in a general setting. The main task of finding interesting examples of deformation maps is taken up in Section 3. Here we consider a simple class of such mappings $\rho$, given by sequences of $n$-point functions, and their compatible states. We show that by carefully adjusting these $n$-point functions, one arrives at an infinite class of deformations, leading to new Poincaré covariant and wedge-local model theories in any number of space-time dimensions. These models are investigated in more detail in Section 4 where Hilbert space representations of deformed quantum fields are presented, and it is shown that they describe non-trivial interaction. The two-particle S-matrix can be calculated explicitly, and depends on the deformation parameter.

The representing quantum fields are typically unbounded operators. In Section 5 we show how to pass from these fields to associated von Neumann algebras, and analyze their TomitaTakesaki modular structure.

In Section 6, we consider the special case of two-dimensional Minkowski space. Here our construction yields a known family of completely integrable quantum field theories. It is shown that the structure of the deformation maps implies characteristic features of the S-matrix, such as its analyticity and crossing properties. Section 7 contains our conclusions.

\section{Deformation maps on the Borchers-Uhlmann algebra}

In this section, we formulate a general deformation scenario for Wightman quantum field theories, based on the tensor algebra $\mathscr{\mathscr { S }}$ over the Schwartz space $\mathscr{S}\left(\mathbb{R}^{d}\right)$. We will assume that the space-time dimension $d \geq 1+1$ is even, as this slightly simplifies our discussion in some places. Most of the following can also be formulated in a vastly more general setting of quite 
general topological *-algebras, but since the examples to be discussed later make use of the specific structure of $\mathscr{\mathscr { S }}$, we restrict our considerations to this particular algebra also in this section.

Let us first recall the structure of the Borchers-Uhlmann algebra $\mathscr{S}$ Bor62, Uhl62]: As a topological vector space, $\mathscr{\mathscr { S }}=\bigoplus_{n=0}^{\infty} \mathscr{S}_{n}$ is the locally convex direct sum of the Schwartz spaces $\mathscr{S}_{n}:=\mathscr{S}\left(\mathbb{R}^{n d}\right), n \geq 0$, with $\mathscr{S}_{0}:=\mathbb{C}$. Elements of $\mathscr{\mathscr { S }}$ are thus terminating sequences $f=\left(f_{0}, f_{1}, f_{2}, \ldots, f_{N}, 0, \ldots\right), f_{n} \in \mathscr{S}_{n}$. Equipped with the tensor product

$$
(f \otimes g)_{n}\left(x_{1}, \ldots, x_{n}\right):=\sum_{k=0}^{n} f_{k}\left(x_{1}, \ldots, x_{k}\right) \cdot g_{n-k}\left(x_{k+1}, \ldots, x_{n}\right), \quad x_{1}, \ldots, x_{n} \in \mathbb{R}^{d},
$$

*-involution

$$
f^{*}{ }_{n}\left(x_{1}, \ldots, x_{n}\right):=\overline{f_{n}\left(x_{n}, \ldots, x_{1}\right)},
$$

and unit $1_{n}:=\delta_{n, 0}$, the linear space $\mathscr{S}$ becomes a unital topological ${ }^{*}$-algebra.

On $\underline{\mathscr{S}}$, the proper orthochronous Poincaré group acts by the continuous automorphisms

$$
\left(\alpha_{a, \Lambda} f\right)_{n}\left(x_{1}, \ldots, x_{n}\right):=f_{n}\left(\Lambda^{-1}\left(x_{1}-a\right), \ldots, \Lambda^{-1}\left(x_{n}-a\right)\right), \quad(a, \Lambda) \in \mathcal{P}_{+}^{\uparrow} .
$$

For our purposes, it is advantageous to implement time-reversing Lorentz transformations by antilinear maps on $\mathscr{S}$. In particular, the reflection $j\left(x^{0}, \ldots, x^{d-1}\right):=\left(-x^{0},-x^{1}, x^{2}, \ldots, x^{d-1}\right)$ acts on $\mathscr{\mathscr { S }}$ according to

$$
\left(\alpha_{j} f\right)_{n}\left(x_{1}, \ldots, x_{n}\right):=\overline{f_{n}\left(j x_{1}, \ldots, j x_{n}\right)},
$$

and yields an extension of $\alpha$ to an automorphic action of the proper Poincaré group $\mathcal{P}_{+}$on $\underline{\mathscr{S}}$ (antilinear for $\mathcal{P}_{+}^{\downarrow}$ ).

We define the support supp $f$ of an element $f \in \mathscr{\mathscr { S }}$ as the smallest closed set $O$ in $\mathbb{R}^{d}$ such that $\operatorname{supp} f_{n} \subset O^{\times n}$ for all $n \geq 1$. Given $O \subset \mathbb{R}^{d}$ and $f_{n} \in \mathscr{S}_{n}$, we will also write $\operatorname{supp} f_{n} \subset O$ or $f_{n} \in \mathscr{S}_{n}(O)$ instead of $\operatorname{supp} f_{n} \subset O^{\times n}$. With this definition of support, the set $\mathscr{\mathscr { S }}(O):=\{f \in \underline{\mathscr{S}}: \operatorname{supp} f \subset O\}$ is a unital ${ }^{*}$-subalgebra of $\mathscr{\mathscr { S }}$, for any $O \subset \mathbb{R}^{d}$. Since $\operatorname{supp} \alpha_{x, \Lambda}(f)=\Lambda \operatorname{supp} f+x$, the automorphisms $\alpha_{x, \Lambda}$ act covariantly on the net $O \mapsto \underline{\mathscr{S}}(O)$,

$$
\alpha_{x, \Lambda}(\underline{\mathscr{S}}(O))=\underline{\mathscr{S}}(\Lambda O+x) .
$$

This net becomes local, i.e., subalgebras $\underline{\mathscr{S}}\left(O_{1}\right), \underline{\mathscr{S}}\left(O_{2}\right) \subset \mathscr{\mathscr { S }}$ associated with spacelike separated regions $O_{1} \subset O_{2}^{\prime}$ commute, after dividing by the so-called locality ideal [Bor62, Yng84, the two-sided ideal $\mathscr{L} \subset \mathscr{S}$ generated by all commutators $f_{1} \otimes g_{1}-g_{1} \otimes f_{1}$, with $f_{1}, g_{1} \in \mathscr{S}_{1}$ having spacelike separated supports.

We will consider states on $\mathscr{L}$ subsequently, and introduce here some notation regarding GNS representations. For a state $\omega$ on $\underline{\mathscr{S}}$, we write $\left(\mathcal{H}_{\omega}, \phi_{\omega}, \Omega_{\omega}\right)$ for the GNS triple associated with $(\underline{\mathscr{S}}, \omega)$, and $\mathcal{D}_{\omega}:=\phi_{\omega}(\underline{\mathscr{S}}) \Omega_{\omega} \subset \mathcal{H}_{\omega}$ for the (dense) domain of the representing field operators. The equivalence classes $\left\{f+g \in \underline{\mathscr{S}}: \omega\left(g^{*} \otimes g\right)=0\right\}$ will be denoted $\Psi_{\omega}(f) \in \mathcal{D}_{\omega}$. Thus $\Psi_{\omega}(1)=\Omega_{\omega}$, and the fields act on $\mathcal{D}_{\omega}$ according to $\phi_{\omega}(f) \Psi_{\omega}(g)=\Psi_{\omega}(f \otimes g)$. As $\phi_{\omega}$ is a representation, we have $\phi_{\omega}(f) \phi_{\omega}(g)=\phi_{\omega}(f \otimes g)$ and $\phi_{\omega}(f)^{*} \supset \phi_{\omega}\left(f^{*}\right)$. The represented localized field algebras are denoted $\mathscr{P}_{\omega}(O):=\phi_{\omega}(\underline{\mathscr{S}}(O)), O \subset \mathbb{R}^{d}$.

Quantum field theories arise from the tensor algebra $\mathscr{L}$ as GNS-representations in suitable states [SW64]. For a state $\omega$ which vanishes on the locality ideal $\mathscr{L}$, field operators $\phi_{\omega}(f)$ and 
$\phi_{\omega}(g)$ commute on $\mathcal{D}_{\omega}$ if the supports of $f$ and $g$ are spacelike separated. If $\omega$ is also invariant under the automorphisms $\alpha_{x, \Lambda}$ (invariant up to a conjugation for time-reversing $\Lambda$ ), there also exists an (anti-)unitary representation $U_{\omega}$ of the proper Poincare group on $\mathcal{H}_{\omega}$ which implements the automorphisms $\alpha_{x, \Lambda}$. In this case, we obtain the familiar structure of a covariant net of local *-algebras:

$$
\begin{gathered}
\mathscr{P}_{\omega}\left(O_{1}\right) \subset \mathscr{P}_{\omega}\left(O_{2}\right) \text { for } O_{1} \subset O_{2}, \\
U_{\omega}(x, \Lambda) \mathscr{P}_{\omega}(O) U_{\omega}(x, \Lambda)^{-1}=\mathscr{P}_{\omega}(\Lambda O+x), \\
{\left[\mathscr{P}_{\omega}\left(O_{1}\right), \mathscr{P}_{\omega}\left(O_{2}\right)\right] \mathcal{D}_{\omega}=0 \text { for } O_{1} \subset O_{2}^{\prime},}
\end{gathered}
$$

where $O_{2}^{\prime}$ denotes the causal complement of $O_{2}$ in $\mathbb{R}^{d}$. For vacuum states, one is interested in the situation where the translations $x \mapsto U_{\omega}(x, 1)$ fulfill the spectrum condition. In this case, also a Reeh-Schlieder property holds, i.e., the subspace $\mathscr{P}_{\omega}(O) \Omega_{\omega}$ is dense in $\mathcal{H}_{\omega}$ for any open region $O \subset \mathbb{R}^{d}$.

On a technical level, note that the field operators $\phi_{\omega}(f), f \in \underline{\mathscr{S}}$, are densely defined on the common $U_{\omega}$-invariant domain $\mathcal{D}_{\omega}$ and closable, but in general unbounded. Several conditions on $\omega$ are known which imply that one can pass from such a net of unbounded operators to nets of von Neumann algebras on $\mathcal{H}_{\omega}$ [BZ63, DSW86, Buc90, BY90. We will however not deal with this question here, and consider only algebras of unbounded operators.

The construction of states which annihilate $\mathscr{L}$ and satisfy the spectrum condition has proven to be extremely difficult. In more than two space-time dimensions, only states leading to (generalized) free field theories are known. In view of these difficulties, we will not attempt a direct construction of quantum field theories by finding suitable states $\omega$ on $\underline{\mathscr{S}}$, but rather use a deformation approach.

To explain this approach, we first recall that in the construction of many models discussed in the recent literature [Bor92, BGL02, Lec03, LR04, BS07, GL07, Lec08, BLS10, DT10, Mun10, a specific weakened version of the net structure (2.5) plays a prominent role. Instead of algebras $\mathscr{P}_{\omega}(O)$ associated with arbitrarily small spacetime regions $O$, one considers only specific regions, so-called wedges. Recall that the right wedge is the region $W_{0}:=\left\{\left(x^{0}, \ldots, x^{d-1}\right) \in\right.$ $\left.\mathbb{R}^{d}: x^{1}>\left|x^{0}\right|\right\}$, and the set $\mathcal{W}$ of all wedges is the Poincaré orbit of $W_{0}$, i.e., $\mathcal{W}:=$ $\left\{\Lambda W_{0}+x:(x, \Lambda) \in \mathcal{P}_{+}\right\}$. In particular, the causal complement $W^{\prime}$ of a wedge $W \in \mathcal{W}$ is also contained in $\mathcal{W}$, and for our reference region $W_{0}$, there holds $W_{0}^{\prime}=-W_{0}=j W_{0}$, with $j\left(x^{0}, \ldots, x^{d-1}\right):=\left(-x^{0},-x^{1}, x^{2}, \ldots, x^{d-1}\right)$ the reflection at the edge of $W_{0}$.

In the context of the GNS data $\mathcal{D}_{\omega} \subset \mathcal{H}_{\omega}, U_{\omega}$ described before, a wedge-local quantum field theory is defined to be a collection of *algebras $\mathscr{P}_{\omega}(W), W \in \mathcal{W}$, consisting of operators defined on $\mathcal{D}_{\omega}$, such that the properties (2.5) hold for $O, O_{1}, O_{2} \in \mathcal{W}$. Since $\mathcal{W}$ consists only of a single Poincaré orbit, such a net can be equivalently characterized in terms of a single algebra [BS08, BW92] $\mathscr{P}_{0}$ of operators acting on $\mathcal{H}_{\omega}$ by requiring

$$
\begin{aligned}
U_{\omega}(x, \Lambda) \mathscr{P}_{0} U_{\omega}(x, \Lambda)^{-1} & \subset \mathscr{P}_{0} \text { for } \Lambda W_{0}+x \subset W_{0}, \\
{\left[U_{\omega}(0, j) \mathscr{P}_{0} U_{\omega}(0, j), \mathscr{P}_{0}\right] \mathcal{D}_{\omega} } & =0 .
\end{aligned}
$$

It is then straightforward to verify that $\mathscr{P}\left(\Lambda W_{0}+x\right):=U(x, \Lambda) \mathscr{P}_{0} U(x, \Lambda)^{-1}$ defines a wedgelocal quantum field theory (A simple causal net in the terminology of [BW92]).

Clearly any net $O \mapsto \mathscr{P}_{\omega}(O)(2.5)$ also defines such a wedge algebra $\mathscr{P}_{0}$. But, as we shall see, an algebra $\mathscr{P}_{0}$ satisfying the conditions (2.6) and (2.7) with respect to a given representation $U_{\omega}$ of $\mathcal{P}_{+}$is much easier to construct than a full net (2.5). Moreover, after 
passing to a net of von Neumann algebras, one can in principle extract algebras of observables localized in arbitrary spacetime regions from these data [BS08, Bor92, BL04.

In the deformation approach, one takes the point of view that a fully local and covariant quantum field theory in the sense of (2.5) is given. These data will usually be realized by free field theories, and in particular define an operator algebra $\mathscr{P}_{0}$ and a representation $U_{\omega}$ in a suitable relative position on some Hilbert space $\mathcal{H}_{\omega}$. One then keeps $\mathcal{H}_{\omega}$ and $U_{\omega}$ fixed, and changes (deforms) the algebra $\mathscr{P}_{0}$ in such a manner that (2.6) and (2.7) remain valid. For suitably chosen deformations, this process leads to inequivalent nets, and in particular turns interaction-free theories into models with non-trivial interaction.

To find examples of deformations preserving the two conditions (2.6) and (2.7), one possible approach is to take the point of view that a deformation of an algebra is a deformation of the product of that algebra. This is the approach taken in the deformation theory of algebras in the mathematics literature [Ger64], which has already led to deformations of quantum field theories in certain examples [GL08, BS08, BLS10].

By a product on $\mathscr{\mathscr { S }}$, we will always mean a bilinear separately continuous map $f, g \mapsto f \hat{\otimes} g$, which is associative and moreover compatible with the unit and star involution in $\mathscr{\mathscr { S }}$, i.e.,

$$
\begin{aligned}
f \hat{\otimes} 1 & =f=1 \hat{\otimes} f, \quad f \in \underline{\mathscr{S}}, \\
(f \hat{\otimes} g)^{*} & =g^{*} \hat{\otimes} f^{*}, \quad f, g \in \underline{\mathscr{S}} .
\end{aligned}
$$

The structure of the family of such products clearly depends on the structure of the algebra under consideration. In the situation at hand, where $\mathscr{S}$ is a tensor algebra, it is known that $\mathscr{\mathscr { S }}$ is rigid in the sense of algebraic deformation theory [Ger64]. That is, all products $\hat{\otimes}$ on $\underline{\mathscr{S}}$ are of the form

$$
f \hat{\otimes} g=\rho^{-1}(\rho(f) \otimes \rho(g))=: f \otimes_{\rho} g,
$$

where $\rho: \underline{\mathscr{S}} \rightarrow \underline{\mathscr{L}}$ is a linear homeomorphism with $\rho(1)=1$ and $\rho(f)^{*}=\rho\left(f^{*}\right)$. Clearly, the algebra $\underline{\mathscr{S}}^{\rho}:=\left(\underline{\mathscr{S}}, \otimes_{\rho}\right)$ given by the linear space $\underline{\mathscr{S}}$, endowed with the product $\otimes_{\rho}$, and unchanged unit and involution, is isomorphic as a unital *-algebra to $\mathscr{L}$. This is the reason why products of the form (2.10) are considered trivial in the deformation theory of single algebras Ger64, and $\mathscr{S}$ is rigid. But we will see later that the use of such trivial deformations will result in non-trivial deformations of nets of wedge algebras nonetheless, as also the local structure of $\underline{\mathscr{S}}$ matters here.

For deformations compatible with localization in wedges, the invariance property (2.6) suggests to require a certain amount of compatibility between the deformation map $\rho$ and the Poincaré action $\alpha$. We therefore make the following definition.

Definition 2.1 A deformation map (relative to $W_{0}$ ) is a linear homeomorphism $\rho: \underline{\mathscr{S}} \rightarrow \underline{\mathscr{S}}$ such that

i) $\rho(1)=1$.

ii) $\rho(f)^{*}=\rho\left(f^{*}\right), f \in \underline{\mathscr{S}}$.

iii) $\rho \circ \alpha_{x, \Lambda}=\alpha_{x, \Lambda} \circ \rho$ for all $(x, \Lambda) \in \mathcal{P}_{+}$with $\Lambda W_{0}+x \subset W_{0}$.

We remark that the third condition in this definition is equivalent to

$$
\rho \circ \alpha_{x, \Lambda}=\alpha_{x, \Lambda} \circ \rho \quad \text { for all } x \in \mathbb{R}^{d} \text { and all } \Lambda \text { with } \Lambda W_{0}=W_{0} .
$$


This is due to the special form of the wedge regions: First, there holds $W_{0}+x \subset W_{0}$ for all $x \in \overline{W_{0}}$. Hence $\alpha_{x} \circ \rho=\rho \circ \alpha_{x}$ for all $x \in \overline{W_{0}}$. Multiplying by $\alpha_{-x}$ from both sides, we see that this equation also holds for $x \in-\overline{W_{0}}$. As any $y \in \mathbb{R}^{d}$ can be written as $y=x+x^{\prime}$ with $x \in \overline{W_{0}}$ and $x^{\prime} \in-\overline{W_{0}}$, this implies that $\rho$ must commute with all translations. Second, if a Poincaré transformation $(x, \Lambda)$ maps $W_{0}$ inside itself, then necessarily $\Lambda W_{0}=W_{0}$ [TW97]. This explains the equivalence of (2.11) with Definition 2.1 iii). To summarize, a deformation map has to preserve the linear, topological, unital, and ${ }^{*}$-structure of $\mathscr{\mathscr { S }}$, and commute with the automorphisms $\alpha_{x, \Lambda}$ for $(x, \Lambda)$ in a specific subgroup of $\mathcal{P}_{+}$, which models the geometry of the wedge region $W_{0}$.

The properties required in Definition 2.1 are stable under composition and taking inverses. With identity as the identity map on $\mathscr{S}$, the deformation maps therefore form a group $\mathcal{R}$. In deformation theory, one is usually interested in studying certain one-parameter families $\rho_{\lambda} \in \mathcal{R}$, $\lambda \in \mathbb{R}$, such that $\lambda \mapsto \rho_{\lambda}$ is continuous in an appropriate sense, and $\rho_{0}=\mathrm{id}$. We will see examples of such one parameter families in Section 3 . For the present general considerations, it will be sufficient to consider deformation maps as such, without introducing a deformation parameter.

Given any deformation map, the product (2.10) will be referred to as the associated deformed product on $\mathscr{\mathscr { S }}$. In view of Definition 2.1 iii), the maps $\alpha_{\Lambda}$ with $\Lambda W_{0}=W_{0}$ act as automorphisms also with respect to the product $\otimes_{\rho}$. For general $\Lambda \in \mathcal{L}_{+}$, one has $\alpha_{\Lambda}\left(f \otimes_{\rho} g\right)=\alpha_{\Lambda}(f) \otimes_{\rho_{\Lambda}} \alpha_{\Lambda}(g)$, where $\rho_{\Lambda}:=\alpha_{\Lambda} \circ \rho \circ \alpha_{\Lambda}^{-1}$ is a deformation map relative to $\Lambda W_{0}$, and in general $\rho_{\Lambda} \neq \rho$. We therefore obtain a whole family of products $\otimes_{\rho_{\Lambda}}$, parametrized by the Lorentz group modulo the stabilizer group of the wedge. This family includes in particular the opposite deformation map

$$
\rho^{\prime}:=\alpha_{j} \circ \rho \circ \alpha_{j}
$$

To construct a wedge-local quantum field theory from this family of deformed products, we have to represent all the algebras $\left(\underline{\mathscr{L}}, \otimes_{\rho_{\Lambda}}\right), \Lambda \in \mathcal{L}_{+}$, on a common Hilbert space. This is possible in specific GNS representations.

Definition 2.2 A state $\omega$ on $\mathscr{S}$ is called compatible with a deformation map $\rho$ if

$$
\omega\left(f \otimes_{\rho} g\right)=\omega(f \otimes g), \quad f, g \in \underline{\mathscr{S}} .
$$

Note that this definition does not imply that multiple deformed products reduce to undeformed products in $\omega$, i.e., in general $\omega\left(f_{1} \otimes_{\rho} \ldots \otimes_{\rho} f_{n}\right) \neq \omega\left(f_{1} \otimes \ldots \otimes f_{n}\right)$ for $n>2$. We are interested in compatible states because they produce common representation spaces for deformed and undeformed tensor products via the GNS construction.

Proposition 2.3 Let $\rho$ be a deformation map and $\omega$ a $\rho$-compatible state. Then $\omega$ is also a state on $\underline{\mathscr{L}}^{\rho}$, and the $G N S$ triples $\left(\mathcal{H}_{\omega}, \phi_{\omega}, \Omega_{\omega}\right)$ of $(\underline{\mathscr{L}}, \omega)$ and $\left(\mathcal{H}_{\omega}^{\rho}, \phi_{\omega}^{\rho}, \Omega_{\omega}^{\rho}\right)$ of $\left(\underline{\mathscr{L}}^{\rho}, \omega\right)$ are related by

$$
\begin{aligned}
\mathcal{H}_{\omega}^{\rho} & =\mathcal{H}_{\omega}, \\
\Omega_{\omega}^{\rho} & =\Omega_{\omega}, \\
\phi_{\omega}^{\rho}(f) \phi_{\omega}(g) \Omega_{\omega} & =\phi_{\omega}\left(f \otimes_{\rho} g\right) \Omega_{\omega}, \quad f, g \in \underline{\mathscr{S}} .
\end{aligned}
$$


Proof: The state $\omega$ on $\mathscr{S}$ clearly defines a normalized linear functional $f \mapsto \omega(f)$ on $\underline{\mathscr{S}}^{\rho}$. The $\rho$-compatibility and positivity of $\omega$ imply, $f \in \underline{\mathscr{S}}$,

$$
\omega\left(f^{*} \otimes_{\rho} f\right)=\omega\left(f^{*} \otimes f\right) \geq 0 .
$$

Hence $\omega$ is also a state on the deformed algebra $\underline{\mathscr{L}}^{\rho}$.

To verify the statements about the GNS representations of $(\mathscr{S}, \omega)$ and $\left(\underline{\mathscr{S}}^{\rho}, \omega\right)$, let $\mathscr{N}_{\omega}:=$ $\left\{f \in \mathscr{\mathscr { S }}: \omega\left(f^{*} \otimes f\right)=0\right\}$ and $\mathscr{N}_{\omega}^{\rho}:=\left\{f \in \underline{\mathscr{S}}: \omega\left(f^{*} \otimes_{\rho} f\right)=0\right\}$ denote the respective Gelfand ideals. Since $\omega$ is $\rho$-compatible, we have $\omega\left(f^{*} \otimes_{\rho} f\right)=\omega\left(f^{*} \otimes f\right)$, and hence $\mathscr{N}_{\omega}^{\rho}=\mathscr{N}_{\omega}$ as linear spaces. As also $\underline{\mathscr{S}}$ and $\underline{\mathscr{S}}^{\rho}$ coincide as linear spaces, we have $\underline{\mathscr{L}} / \mathscr{N}_{\omega}=\mathscr{\mathscr { S }}^{\rho} / \mathscr{N}_{\omega}^{\rho}$. By the $\rho$-compatability of $\omega$, these pre-Hilbert spaces carry the same scalar product $\left\langle\Psi_{\omega}(f), \Psi_{\omega}(g)\right\rangle=$ $\omega\left(f^{*} \otimes g\right)=\omega\left(f^{*} \otimes_{\rho} g\right)$, which implies in particular that their Hilbert space closures $\mathcal{H}_{\omega}$ and $\mathcal{H}_{\omega}^{\rho}$ are identical. The implementing vectors $\Omega_{\omega}$ and $\Omega_{\omega}^{\rho}$ are both equal to the equivalence class $\Psi_{\omega}(1)=\Psi_{\omega}^{\rho}(1)$ and therefore identical.

The GNS representation $\phi_{\omega}^{\rho}$ of $\underline{\mathscr{S}}^{\rho}$ acts on this space according to, $f, g \in \underline{\mathscr{S}}$,

$$
\phi_{\omega}^{\rho}(f) \phi_{\omega}(g) \Omega_{\omega}=\phi_{\omega}^{\rho}(f) \Psi_{\omega}(g)=\Psi_{\omega}\left(f \otimes_{\rho} g\right)=\phi_{\omega}\left(f \otimes_{\rho} g\right) \Omega_{\omega} .
$$

This is well-defined since $\mathscr{N}_{\omega}$ is, by the preceding argument, also a left ideal with respect to the deformed product, and the proof is finished.

We now explain how wedge-local quantum field theories can be constructed from deformation maps $\rho$. To this end, suppose $\rho$ is a deformation map, and $\omega$ is a $\rho$-compatible state which is invariant under $\alpha$ in the sense that, $f \in \underline{\mathscr{S}}$,

$$
\omega\left(\alpha_{x, \Lambda}(f)\right)=\left\{\begin{array}{lll}
\overline{\omega(f)} & ; \quad(x, \Lambda) \in \mathcal{P}_{+}^{\downarrow} \\
\omega(f) & ; \quad(x, \Lambda) \in \mathcal{P}_{+}^{\uparrow}
\end{array} .\right.
$$

We then have, $f, g \in \underline{\mathscr{L}}, \Lambda \in \mathcal{L}_{+}^{\uparrow}$,

$$
\begin{aligned}
\omega\left(f \otimes_{\rho_{\Lambda}} g\right) & =\omega\left(\alpha_{\Lambda}\left(\alpha_{\Lambda}^{-1}(f) \otimes_{\rho} \alpha_{\Lambda}^{-1}(g)\right)\right)=\omega\left(\alpha_{\Lambda}^{-1}(f) \otimes_{\rho} \alpha_{\Lambda}^{-1}(g)\right) \\
& =\omega\left(\alpha_{\Lambda}^{-1}(f) \otimes \alpha_{\Lambda}^{-1}(g)\right)=\omega(f \otimes g),
\end{aligned}
$$

and by an analogous calculation, also $\omega\left(f \otimes_{\rho_{\Lambda}} g\right)=\omega(f \otimes g)$ for $\Lambda \in \mathcal{L}_{+}^{\downarrow}$. Hence the state $\omega$ is compatible with all Lorentz transformed deformation maps $\rho_{\Lambda}, \Lambda \in \mathcal{L}_{+}$. In view of Proposition 2.3, all these deformations are thus realized on the GNS space of the undeformed algebra, and can be compared in terms of the Hilbert space operators $\phi_{\omega}^{\rho_{\Lambda}}(f)$ on $\mathcal{D}_{\omega} \subset \mathcal{H}_{\omega}$.

It is clear from our construction that the (anti-)unitary representation $U_{\omega}$ implementing $\alpha$ on $\mathcal{H}_{\omega}$ satisfies $U_{\omega}(x, \Lambda) \Psi_{\omega}(f)=\Psi_{\omega}\left(\alpha_{x, \Lambda} f\right),(x, \Lambda) \in \mathcal{P}_{+}, f \in \underline{\mathscr{S}}$. After a small calculation making use of $\phi_{\omega}^{\rho_{\Lambda}}(f) \Psi_{\omega}(g)=\Psi_{\omega}\left(f \otimes_{\rho_{\Lambda}} g\right)$ (2.16), this yields the transformation law

$$
U_{\omega}(x, \Lambda) \phi_{\omega}^{\rho}(f) U_{\omega}(x, \Lambda)^{-1}=\phi_{\omega}^{\rho_{\Lambda}}\left(\alpha_{x, \Lambda} f\right), \quad(x, \Lambda) \in \mathcal{P}_{+}, f \in \underline{\mathscr{S}} .
$$

In particular, for those transformations $(x, \Lambda)$ that satisfy $\Lambda W_{0}+x \subset W_{0}$, the corresponding automorphisms commute with $\rho$ (Definition 2.1 iii)), and we have

$$
U_{\omega}(x, \Lambda) \phi_{\omega}^{\rho}(f) U_{\omega}(x, \Lambda)^{-1}=\phi_{\omega}^{\rho}\left(\alpha_{x, \Lambda} f\right), \quad \Lambda W_{0}+x \subset W_{0}, f \in \underline{\mathscr{S}} .
$$

To produce a wedge-localized algebra complying with (2.6) and (2.7), we have to use elements $f \in \underline{\mathscr{S}}$ with support in $W_{0}$. As the deformation map $\rho$ will usually not preserve supports, 
$\underline{\mathscr{S}}\left(W_{0}\right)$ will not be an algebra with respect to the deformed product $\otimes_{\rho}$. We therefore consider the *-algebra $\mathscr{P}_{\omega, 0}^{\rho}$ generated by all $\phi_{\omega}^{\rho}(f), f \in \underline{\mathscr{S}}\left(W_{0}\right)$. The transformation law (2.20) then implies the desired invariance (2.6) of $\mathscr{P}_{\omega, 0}^{\rho}$.

The crucial locality condition (2.7) is equivalent to the vanishing of the commutators

$$
\left[\phi_{\omega}^{\rho}(f), \phi_{\omega}^{\rho^{\prime}}\left(g^{\prime}\right)\right] \Psi=0, \quad f \in \underline{\mathscr{S}}\left(W_{0}\right), g \in \underline{\mathscr{S}}\left(W_{0}^{\prime}\right), \Psi \in \mathcal{D}_{\omega} .
$$

We will say that a deformation map $\rho$ is wedge-local in a state $\omega$ which is compatible with $\rho$ and $\rho^{\prime}$ if (2.21) holds. In this case, the algebra $\mathscr{P}_{\omega, 0}^{\rho}$ complies with (2.6) and (2.7), and can therefore be used to generate a quantum field theory model.

To illustrate the conditions on the interplay of $\rho$ and $\omega$, we recall that the deformation map given by warped convolution GL08 is compatible with all translationally invariant states on $\mathscr{S}$. But the locality condition (2.21) is only valid if $\omega$ annihilates $\mathscr{L}$, the translations $U_{\omega}(x, 1)$ satisfy a spectrum condition, and the parameters defining $\rho$ are suitably chosen [GL08, BLS10]. Hence the validity of (2.21) is not a property of $\rho$ alone, but also involves properties of $\omega$ going beyond compatibility and vanishing on $\mathscr{L}$.

In the present generality, it seems to be difficult to find manageable conditions on $\rho$ and $\omega$ which imply that $\rho$ is wedge-local in $\omega$. We will therefore present in the next section a family of explicit deformation maps $\rho$ together with their compatible states $\omega$ such that $\rho$ is wedgelocal in $\omega$. Before moving on to the examples, we point out that the wedge-locality condition amounts to the vanishing of matrix elements of commutators with respect to the undeformed product, a result that will be useful later on.

Lemma 2.4 Let $\rho$ be a deformation map and $\omega$ a state on $\underline{\mathscr{S}}$ which is compatible with $\rho$ and $\rho^{\prime}$. Then $\rho$ is wedge-local in $\omega$ if and only if

$$
\omega\left(\left(h \otimes_{\rho} f\right) \otimes\left(g^{\prime} \otimes_{\rho^{\prime}} k\right)\right)=\omega\left(\left(h \otimes_{\rho^{\prime}} g^{\prime}\right) \otimes\left(f \otimes_{\rho} k\right)\right)
$$

for all $f \in \underline{\mathscr{S}}\left(W_{0}\right), g^{\prime} \in \underline{\mathscr{S}}\left(W_{0}^{\prime}\right), h, k \in \underline{\mathscr{S}}$.

Proof: For $\rho$ to be wedge-local in $\omega$, we need to show $\left[\phi_{\omega}^{\rho}(f), \phi_{\omega}^{\rho^{\prime}}\left(g^{\prime}\right)\right] \Psi=0$ for all $\Psi \in \mathcal{D}_{\omega}, f \in$ $\underline{\mathscr{S}}\left(W_{0}\right), g^{\prime} \in \mathscr{\mathscr { S }}\left(W_{0}^{\prime}\right)$. Since $\mathcal{D}_{\omega}=\phi_{\omega}(\underline{\mathscr{S}}) \Omega_{\omega}$ is dense in $\mathcal{H}_{\omega}$, this is equivalent to the vanishing of the matrix elements $\left\langle\Omega_{\omega}, \phi_{\omega}(h)\left[\phi_{\omega}^{\rho}(f), \phi_{\omega}^{\rho^{\prime}}\left(g^{\prime}\right)\right] \phi_{\omega}(k) \Omega_{\omega}\right\rangle=0$ for arbitrary $h, k \in \underline{\mathscr{S}}$. But in view of the compatibility of $\rho, \rho^{\prime}$ with $\omega$, and the associativity of the products $\otimes_{\rho}, \otimes_{\rho^{\prime}}$, we can rewrite these matrix elements as

$$
\begin{aligned}
0 & =\left\langle\Omega_{\omega}, \phi_{\omega}(h)\left[\phi_{\omega}^{\rho}(f), \phi_{\omega}^{\rho^{\prime}}\left(g^{\prime}\right)\right] \phi_{\omega}(k) \Omega_{\omega}\right\rangle \\
& =\left\langle\Omega_{\omega}, \phi_{\omega}(h) \phi_{\omega}\left(f \otimes_{\rho}\left(g^{\prime} \otimes_{\rho^{\prime}} k\right)\right) \Omega_{\omega}\right\rangle-\left\langle\Omega_{\omega}, \phi_{\omega}(h) \phi_{\omega}\left(g^{\prime} \otimes_{\rho^{\prime}}\left(f \otimes_{\rho} k\right)\right) \Omega_{\omega}\right\rangle \\
& =\omega\left(h \otimes\left(f \otimes_{\rho}\left(g^{\prime} \otimes_{\rho^{\prime}} k\right)\right)\right)-\omega\left(h \otimes\left(g^{\prime} \otimes_{\rho^{\prime}}\left(f \otimes_{\rho} k\right)\right)\right) \\
& =\omega\left(h \otimes_{\rho} f \otimes_{\rho}\left(g^{\prime} \otimes_{\rho^{\prime}} k\right)\right)-\omega\left(h \otimes_{\rho^{\prime}} g^{\prime} \otimes_{\rho^{\prime}}\left(f \otimes_{\rho} k\right)\right) \\
& =\omega\left(\left(h \otimes_{\rho} f\right) \otimes_{\rho}\left(g^{\prime} \otimes_{\rho^{\prime}} k\right)\right)-\omega\left(\left(h \otimes_{\rho^{\prime}} g^{\prime}\right) \otimes_{\rho^{\prime}}\left(f \otimes_{\rho} k\right)\right) \\
& \left.=\omega\left(\left(h \otimes_{\rho} f\right) \otimes^{\prime} \otimes_{\rho^{\prime}} k\right)\right)-\omega\left(\left(h \otimes_{\rho^{\prime}} g^{\prime}\right) \otimes\left(f \otimes_{\rho} k\right)\right) .
\end{aligned}
$$

As the last expression is identical to (2.22), the proof is finished. 


\section{Multiplicative deformations and their compatible states}

We now turn to the task of finding examples of deformation maps $\rho$ which meet our requirements. It will be convenient to work in momentum space most of the time, i.e., we consider the Fourier transform $f \mapsto \tilde{f}$ on $\underline{\mathscr{L}}$,

$$
\tilde{f}_{n}\left(p_{1}, \ldots, p_{n}\right):=(2 \pi)^{-n d / 2} \int d^{d} x_{1} \cdots d^{d} x_{n} f_{n}\left(x_{1}, \ldots, x_{n}\right) e^{i p_{1} \cdot x_{1}} \cdots e^{i p_{n} \cdot x_{n}} .
$$

This map preserves the linear and product structure of $\mathscr{\mathscr { S }}$ as well as its identity element. Furthermore, the Fourier transform commutes with the action of the orthochronous Lorentz transformations, and thus $\mathcal{L}_{+}^{\uparrow}$ acts on the momentum space wave functions in the same manner as in (2.3). Translations, the ${ }^{*}$-involution, and the reflection at the edge of the wedge take the form, $p_{1}, \ldots, p_{n} \in \mathbb{R}^{d}$,

$$
\begin{aligned}
& \widetilde{\left(\widetilde{\alpha_{x} f}\right)_{n}}\left(p_{1}, \ldots, p_{n}\right)=e^{i\left(p_{1}+\ldots+p_{n}\right) \cdot x} \cdot \tilde{f}_{n}\left(p_{1}, \ldots, p_{n}\right), \\
& \widetilde{f^{*}}{ }_{n}\left(p_{1}, \ldots, p_{n}\right) \\
& \widetilde{\tilde{f}_{n}\left(-p_{n}, \ldots,-p_{1}\right)} \\
&\left(\widetilde{\alpha_{j} f}\right)_{n}\left(p_{1}, \ldots, p_{n}\right)=\overline{\tilde{f}_{n}\left(-j p_{1}, \ldots,-j p_{n}\right)} .
\end{aligned}
$$

After these remarks, we consider deformation maps $\rho: \mathscr{S} \rightarrow \mathscr{S}$ in the sense of Definition 2.1. In view of the structure of $\mathscr{\mathscr { S }}$, every such map is given by a family of (distributional) integral kernels $\rho_{n m}, n, m \in \mathbb{N}_{0}$, such that, $f_{n} \in \mathscr{S}_{n}$,

$$
\widetilde{\rho\left(f_{n}\right)_{m}}\left(p_{1}, \ldots, p_{m}\right)=\int d q_{1} \cdots d q_{n} \rho_{n m}\left(q_{1}, \ldots, q_{n} ; p_{1}, \ldots, p_{m}\right) \tilde{f}_{n}\left(q_{1}, \ldots, q_{n}\right) .
$$

The defining properties of a deformation map restrict the possible form of the distributions $\rho_{n m}$. For example, property iii) of Definition 2.1 requires the support of $\rho_{n m}$ to be contained in $\left\{\left(q_{1}, \ldots, q_{n}, p_{1}, \ldots, p_{m}\right): q_{1}+. .+q_{n}+p_{1}+. .+p_{m}=0\right\}$, similar to the energy-momentum conservation of S-matrix elements.

A systematic study of deformation maps and the emerging deformed quantum field theories will be presented elsewhere. Here we consider a particularly simple class of maps $\rho: \underline{\mathscr{S}} \rightarrow \underline{\mathscr{S}}$ which preserve the grading of $\mathscr{\mathscr { S }}$ and act multiplicatively in momentum space, i.e., are of the form

$$
\widetilde{\rho(f)_{n}}\left(p_{1}, \ldots, p_{n}\right)=\rho_{n}\left(p_{1}, \ldots, p_{n}\right) \cdot \tilde{f}_{n}\left(p_{1}, \ldots, p_{n}\right), \quad n \in \mathbb{N}_{0}, f \in \underline{\mathscr{S}} .
$$

We will refer to deformation maps of this type as multiplicative deformations. They form an abelian subgroup, denoted $\mathcal{R}_{0}$, of the group $\mathcal{R}$ of all deformation maps. Given $\rho \in \mathcal{R}_{0}$, the functions $\rho_{n}$ (3.5) are called the $n$-point functions of $\rho$, and it is straightforward to characterize $\rho$ in terms of the $\rho_{n}$.

Lemma 3.1 The group $\mathcal{R}_{0}$ of multiplicative deformations of $\mathscr{S}$ consists precisely of those sequences $\rho_{n} \in C^{\infty}\left(\mathbb{R}^{n d}\right), n \in \mathbb{N}_{0}$, of smooth functions which satisfy the following conditions.

i) For each multi index $\boldsymbol{\mu} \in \mathbb{N}_{0}^{n d}$, there exist $N_{\boldsymbol{\mu}} \in \mathbb{R}$ and $C_{\boldsymbol{\mu}}>0$ such that

$$
\left|\partial^{\mu} \rho_{n}\left(p_{1}, \ldots, p_{n}\right)\right| \leq C_{\boldsymbol{\mu}}\left(1+\left|p_{1}\right|^{2}+\ldots+\left|p_{n}\right|^{2}\right)^{N_{\mu}}, \quad p_{1}, \ldots, p_{n} \in \mathbb{R}^{d} .
$$

ii) There exists $M \in \mathbb{R}$ and $C^{\prime}>0$ such that

$$
\left|\rho_{n}\left(p_{1}, \ldots, p_{n}\right)\right| \geq C^{\prime}\left(1+\left|p_{1}\right|^{2}+\ldots+\left|p_{n}\right|^{2}\right)^{-M}, \quad p_{1}, \ldots, p_{n} \in \mathbb{R}^{d} .
$$


iii) For each Lorentz transformation $\Lambda$ with $\Lambda W_{0}=W_{0}$,

$$
\rho_{n}\left(\Lambda p_{1}, \ldots, \Lambda p_{n}\right)=\rho_{n}\left(p_{1}, \ldots, p_{n}\right), \quad p_{1}, \ldots, p_{n} \in \mathbb{R}^{d} .
$$

iv) $\rho_{n}$ is ${ }^{*}$-invariant,

$$
\overline{\rho_{n}\left(-p_{n}, \ldots,-p_{1}\right)}=\rho_{n}\left(p_{1}, \ldots, p_{n}\right), \quad p_{1}, \ldots, p_{n} \in \mathbb{R}^{d} .
$$

v) $\rho_{0}=1$.

Proof: The first two conditions i), ii) are necessary and sufficient for $\rho$ to be a homeomorphism on $\mathscr{S}$ : Let us first assume i), ii) hold. Then, by condition i), $f_{n} \mapsto \rho_{n} \cdot f_{n}$ maps $\mathscr{S}_{n}$ into $\mathscr{S}_{n}$. Moreover, this map is linear and it is straightforward to see that it is continuous in the Schwartz topology. By condition ii), the $\rho_{n}$ are in particular non-vanishing, and the reciprocals $1 / \rho_{n}$ are polynomially bounded by (3.7). It now follows by application of the chain rule that all derivatives of $1 / \rho_{n}$ satisfy polynomial bounds of the form (3.6). Hence $f_{n} \mapsto f_{n} / \rho_{n}$ is also a continuous linear map from $\mathscr{S}_{n}$ onto $\mathscr{S}_{n}$, with inverse $\rho_{n}$.

The map $\rho: \underline{\mathscr{S}} \rightarrow \underline{\mathscr{S}}$ on the direct sum $\underline{\mathscr{S}}=\bigoplus_{n} \mathscr{S}_{n}$ is continuous iff its restriction to $\mathscr{S}_{n}$ is continuous for each $n$ [Tre67]. But the restriction of $\rho$ to $\mathscr{S}_{n}$ maps this space continuously onto $\mathscr{S}_{n}$, which in turn is continuously embedded in $\mathscr{\mathscr { L }}$. Hence $\rho$ is continuous, and by the same argument, one sees that $\rho^{-1}$ is continuous as well. Thus $\rho$ is a linear homeomorphism, as required in Definition 2.1.

Conversely, let us now assume that $\rho$ defined as in (3.5) is a homeomorphism of $\underline{\mathscr{L}}$. For such a multiplicative transformation to map $\mathscr{S}_{n}$ onto $\mathscr{S}_{n}$, it is necessary that $\rho_{n}$ is smooth and polynomially bounded in all derivatives, i.e., i) holds. Since $\rho^{-1}$ has the same properties, also ii) follows.

Condition iii) is equivalent to $\alpha_{\Lambda} \circ \rho=\rho \circ \alpha_{\Lambda}$ for $\Lambda$ with $\Lambda W_{0}=W_{0}$, as a short calculation based on (3.5) and (2.3) shows. Translational invariance imposes no further restrictions on $\rho$ since both $\rho$ and the translations act multiplicatively in momentum space and therefore commute automatically.

Using (3.5) and (3.2), one easily checks that iv) is equivalent to $\rho\left(f^{*}\right)=\rho(f)^{*}, f \in \underline{\mathscr{S}}$. Since $\rho(1)_{0}=\rho_{0}$, condition $\left.v\right)$ is equivalent to $\rho(1)=1$.

Remark: For $\rho \in \mathcal{R}_{0}$, the opposite deformation $\rho^{\prime}=\alpha_{j} \circ \rho \circ \alpha_{j}$ is given by the $n$-point functions $\rho_{n}^{\prime}\left(p_{1}, \ldots, p_{n}\right)=\overline{\rho_{n}\left(-j p_{1}, \ldots,-j p_{n}\right)}$. But as the Lorentz transformation $-j$ maps the wedge $W_{0}$ onto itself, and $-j \in \mathcal{L}_{+}^{\uparrow}$ because $d$ is even, we can use the invariance stated in part iii) of Lemma 3.1 to rewrite the $n$-point functions of the opposite deformation as

$$
\rho_{n}^{\prime}\left(p_{1}, \ldots, p_{n}\right)=\overline{\rho_{n}\left(p_{1}, \ldots, p_{n}\right)} .
$$

The inverse $\rho^{-1}$ of a multiplicative deformation $\rho \in \mathcal{R}_{0}$ is given by the reciprocal $n$-point functions $1 / \rho_{n}$, and thus the product $f \otimes_{\rho} g=\rho^{-1}(\rho(f) \otimes \rho(g))$ takes the following simple form in momentum space,

$$
\left(\widetilde{f \otimes_{\rho} g}\right)_{n}\left(p_{1}, . ., p_{n}\right)=\sum_{k=0}^{n} \frac{\rho_{k}\left(p_{1}, . ., p_{k}\right) \rho_{n-k}\left(p_{k+1}, . ., p_{n}\right)}{\rho_{n}\left(p_{1}, . ., p_{n}\right)} \tilde{f}_{k}\left(p_{1}, . ., p_{k}\right) \tilde{g}_{n-k}\left(p_{k+1}, . ., p_{n}\right) .
$$


It is clear from the conditions spelled out in Lemma 3.1 that many multiplicative deformation maps exist. However, different $\rho, \hat{\rho} \in \mathcal{R}_{0}$ might induce the same product (3.9) on $\mathscr{L}$. We therefore introduce an equivalence relation on $\mathcal{R}_{0}$ by defining $\rho, \hat{\rho}$ as equivalent, in symbols $\rho \sim \hat{\rho}$, if $f \otimes_{\rho} g=f \otimes_{\hat{\rho}} g$ for all $f, g \in \underline{\mathscr{S}}$. A multiplicative deformation $\rho \in \mathcal{R}_{0}$ is called trivial if $\rho \sim$ id.

Lemma $3.2 \quad$ i) Two deformations $\rho, \hat{\rho} \in \mathcal{R}_{0}$ are equivalent if and only if $\hat{\rho} \rho^{-1}$ is trivial.

ii) A deformation $\rho \in \mathcal{R}_{0}$ is trivial if and only if $\rho_{n}=\rho_{1}^{\otimes n}, n \in \mathbb{N}$.

iii) Let $\rho \in \mathcal{R}_{0}$. Then there exists another $\hat{\rho} \in \mathcal{R}_{0}$ with $\hat{\rho}_{1}=1$ and $\hat{\rho} \sim \rho$.

Proof: i) Assume $\rho \sim \hat{\rho}$. Then $\rho^{-1}(\rho(f) \otimes \rho(g))=\hat{\rho}^{-1}(\hat{\rho}(f) \otimes \hat{\rho}(g))$ for all $f, g \in \underline{\mathscr{S}}$, or, equivalently, $f \otimes_{\hat{\rho} \rho^{-1}} g=\left(\rho \hat{\rho}^{-1}\right)\left(\left(\hat{\rho} \rho^{-1}\right)(f) \otimes\left(\hat{\rho} \rho^{-1}\right)(g)\right)=f \otimes g$. Hence $\hat{\rho} \rho^{-1}$ is trivial. If, on the other hand, $\hat{\rho} \rho^{-1} \sim \mathrm{id}$, then $\left(\rho \hat{\rho}^{-1}\right)\left(\left(\hat{\rho} \rho^{-1}\right)(f) \otimes\left(\hat{\rho} \rho^{-1}\right)(g)\right)=f \otimes g$, and $\rho \sim \hat{\rho}$ follows.

ii) The triviality condition $\rho^{-1}(\rho(f) \otimes \rho(g))=f \otimes g, f, g \in \mathscr{L}$, is satisfied if and only if $\rho$ is an automorphism of $\mathscr{S}$. As $\rho$ is taken to be multiplicative here, it is an automorphism if and only if $\rho_{n}=\rho_{1}^{\otimes n}, n \in \mathbb{N}$.

iii) Let $\rho \in \mathcal{R}_{0}$. Then $\rho_{1}$ satisfies the conditions $i$ )-iv) in Lemma 3.1 for $n=1$, and it is easy to check that for $n \geq 1$, also the functions $\sigma_{n}:=1 / \rho_{1}^{\otimes n}$ comply with these conditions. With $\sigma_{0}:=1$, this defines a multiplicative deformation $\sigma \in \mathcal{R}_{0}$ which is trivial by part ii). According to part $i$ ), $\hat{\rho}:=\sigma \rho$ is equivalent to $\rho$, and $\hat{\rho}_{1}=\rho_{1} / \rho_{1}=1$.

In view of the last statement, the redundancy in describing deformed products of the form (3.9) by $n$-point functions $\rho_{n}$ is precisely taken into account by restricting to multiplicative deformations $\rho \in \mathcal{R}_{0}$ with trivial one point function $\rho_{1}=1$. We shall therefore consider only such $\rho$ in the following.

Following the general strategy explained in Section 2, we next investigate the compatibility of $\rho \in \mathcal{R}_{0}$ with certain states $\omega$ on $\mathscr{S}$ (Definition 2.2). That is, we need to find physically relevant states such that $\omega\left(f \otimes_{\rho} g\right)=\omega(f \otimes g)$ for all $f, g \in \underline{\mathscr{L}}$. Each state on $\mathscr{\mathscr { L }}$ is given by a sequence of distributions $\omega_{n} \in \mathscr{S}_{n}^{\prime}, n \in \mathbb{N}$, its $n$-point functions, and $\omega_{0}=1$. In momentum space, we have

$$
\omega(f)=\sum_{n=0}^{\infty} \int d p_{1} \cdots d p_{n} \tilde{\omega}_{n}\left(-p_{1}, \ldots,-p_{n}\right) \tilde{f}_{n}\left(p_{1}, \ldots, p_{n}\right), \quad f \in \underline{\mathscr{S}} .
$$

Inserting (3.9) into the condition $\omega\left(f \otimes_{\rho} g\right)=\omega(f \otimes g)$, we observe that if the $\tilde{\omega}_{n}$ are measures, compatibility of $\omega$ with $\rho$ is equivalent to the factorization

$$
\rho_{n}\left(p_{1}, \ldots, p_{n}\right)=\rho_{k}\left(p_{1}, \ldots, p_{k}\right) \cdot \rho_{n-k}\left(p_{k+1}, \ldots, p_{n}\right) \text { for all }\left(p_{1}, \ldots, p_{n}\right) \in-\operatorname{supp} \tilde{\omega}_{n},
$$

for all $n, k \in \mathbb{N}_{0}, k \leq n$. For more singular distributions $\tilde{\omega}_{n}$, compatibility of $\omega$ with $\rho$ poses also conditions on the derivatives of the $\rho_{n}$.

As the momentum space supports of $n$-point functions play a role in the compatibility question, we proceed with some remarks about relevant examples from quantum field theory. A large class of states of interest is the class of all translationally invariant states, satisfying $\omega \circ \alpha_{x}=\omega$ for all $x \in \mathbb{R}^{d}$. Their $n$-point functions have support at zero energy-momentum, that is,

$$
\operatorname{supp} \tilde{\omega}_{n} \subset S_{\text {inv }}^{n}:=\left\{\boldsymbol{p} \in \mathbb{R}^{n d}: p_{1}+\ldots+p_{n}=0\right\} \text {. }
$$


As vacuum states in quantum field theory, one considers the subclass of translationally invariant states satisfying the spectrum condition. These are given by $n$-point functions with Bor62

$$
\operatorname{supp} \tilde{\omega}_{n} \subset S_{\text {Spec }}^{n}:=\left\{\boldsymbol{p} \in \mathbb{R}^{n d}: p_{1}, p_{1}+p_{2}, \ldots, p_{1}+\ldots+p_{n-1} \in \overline{V_{+}}, p_{1}+\ldots+p_{n}=0\right\},
$$

where $\overline{V_{+}}=\left\{q \in \mathbb{R}^{d}: q \cdot q \geq 0, q^{0} \geq 0\right\}$ is the closed forward light cone.

Special examples of states, related to generalized free field models, are given by quasi-free states, which are completely determined by their two-point function $\omega_{2}$. Recall that a state $\omega$ on $\mathscr{\mathscr { S }}$ is called quasi-free if

$$
\tilde{\omega}_{2 n-1}=0, \quad \tilde{\omega}_{2 n}\left(p_{1}, \ldots, p_{2 n}\right)=\sum_{(\boldsymbol{l}, \boldsymbol{r})} \prod_{k=1}^{n} \tilde{\omega}_{2}\left(p_{l_{k}}, p_{r_{k}}\right), \quad n \in \mathbb{N},
$$

where the sum runs over all partitions $(\boldsymbol{l}, \boldsymbol{r})$ of $\{1, \ldots, 2 n\}$ into disjoint tuples $\left(l_{1}, r_{1}\right), \ldots,\left(l_{n}, r_{n}\right)$ with $l_{k}<r_{k}, k=1, \ldots, n$. For quasi-free translationally invariant states $\omega$, we have supp $\tilde{\omega}_{2 n-1}=$ $\emptyset$, and

$$
\operatorname{supp} \tilde{\omega}_{2 n} \subset S_{\mathrm{qf}}^{2 n}:=\bigcup_{(\boldsymbol{l}, \boldsymbol{r})}\left\{\boldsymbol{p} \in \mathbb{R}^{2 n d}: p_{l_{k}}+p_{r_{k}}=0, k=1, \ldots, n\right\}
$$

In view of the positivity $\omega_{2}\left(f_{1}^{*} \otimes f_{1}\right) \geq 0, f_{1} \in \mathscr{S}_{1}$, we can apply Bochner's theorem to conclude that $\tilde{\omega}_{2}$ is a measure. Taking into account the special structure of the $n$-point functions (3.14), it then follows that each $\tilde{\omega}_{n}$ is a measure. In particular, the compatibility of a translationally quasi-free state with a multiplicative deformation is equivalent to the factorization condition (3.11).

Finally, translationally quasi-free states satisfy in addition the spectrum condition if and only if supp $\tilde{\omega}_{2} \subset\left\{(p, q) \in \mathbb{R}^{2 d}: p \in \overline{V_{+}}, p+q=0\right\}$. In this last case, $\omega_{2}$ can be represented as

$$
\tilde{\omega}_{2}(p, q)=\delta(p+q) w(p),
$$

where $w$ is a measure on $\overline{V_{+}}$.

It turns out that it is a very strong condition to require a multiplicative deformation to be compatible with all translationally invariant states, or all translationally invariant states satisfying the spectrum condition. In fact, since there exist sufficiently many such states on $\mathscr{S}$ Yng81, these conditions are equivalent to requiring (3.11) to hold on all of $S_{\text {inv }}^{n}$ (3.12). As a necessary condition for compatibility, this yields a recursive equation determining the $n$-point functions $\rho_{n}, n \geq 2$, in terms of the two point function $\rho_{2}$. In addition, several algebraic relations for $\rho_{2}$ have to be satisfied for (3.11) to hold. One special solution, corresponding to Rieffel deformations and warped convolutions, exists, and will be recalled later on. The most general deformation two-point function complying with these conditions is presently not known, but it seems that there is little freedom for obtaining other deformations $\rho \in \mathcal{R}_{0}$ compatible with all translationally invariant states 1 .

Instead of asking for compatibility of $\rho$ with all translationally invariant states, we will consider in the following the less restrictive condition that $\rho$ should be compatible with all quasi-free translationally invariant states. This amounts to requiring (3.11) to hold on the

\footnotetext{
${ }^{1}$ S. Alazzawi, work in progress.
} 
smaller domain $S_{\mathrm{qf}}^{n}(3.15)$. We will see that an infinite family of such $\rho$ exists, providing non-trivial deformations of generalized free field theories.

Also in the case of multiplicative deformations which are compatible with quasi-free translationally invariant states, the $n$-point functions $\rho_{n}$ are determined by the two-point function $\rho_{2}$. In the following proposition, we show under which conditions on $\rho_{2}$ the required compatibility holds. Explicit solutions of these conditions on $\rho_{2}$ are then discussed in Lemma 3.5.

Proposition 3.3 Let $\rho_{2} \in C^{\infty}\left(\mathbb{R}^{d} \times \mathbb{R}^{d}\right)$ be a two-point function of a multiplicative deformation, satisfying conditions i)-iv) of Lemma 3.1 for $n=2$, and in addition, $p, q \in \mathbb{R}^{d}$,

$$
\rho_{2}(p,-p)=1, \quad \rho_{2}(-p, q)=\rho_{2}(p,-q)=\rho_{2}(q, p)=\rho_{2}(p, q)^{-1} .
$$

Define

$$
\rho_{0}:=1, \quad \rho_{1}\left(p_{1}\right):=1, \quad \rho_{n}\left(p_{1}, \ldots, p_{n}\right):=\prod_{1 \leq l<r \leq n} \rho_{2}\left(p_{l}, p_{r}\right), \quad n \geq 2 .
$$

Then

i) The n-point functions (3.18) define a multiplicative deformation $\rho \in \mathcal{R}_{0}$.

ii) The deformed product associated with $\rho$ has the form

$$
\left.\widetilde{\left(f \otimes_{\rho} g\right.}\right)_{n}\left(p_{1}, . ., p_{n}\right)=\sum_{k=0}^{n}\left(\prod_{l=1}^{k} \prod_{r=k+1}^{n} \rho_{2}\left(p_{l}, p_{r}\right)^{-1}\right) \tilde{f}_{k}\left(p_{1}, . ., p_{k}\right) \tilde{g}_{n-k}\left(p_{k+1}, . ., p_{n}\right) \text {. }
$$

iii) Let $\omega$ be a quasi-free translationally invariant state on $\mathscr{S}$. Then $\rho$ (3.18) and $\rho^{-1}$ are compatible with $\omega$, and for all $f_{n} \in \mathscr{S}_{n}, k \in\{0, \ldots, n\}$, the functions

$$
\tilde{f}_{n, k, \pm}\left(p_{1}, \ldots, p_{n}\right):=\tilde{f}_{n}\left(p_{1}, \ldots, p_{n}\right) \cdot \prod_{l=1}^{k} \prod_{r=k+1}^{n} \rho_{2}\left(p_{l}, p_{r}\right)^{ \pm 1}
$$

have the same expectation value as $f_{n}$ in $\omega$.

iv) The opposite deformation is $\rho^{\prime}=\rho^{-1}$.

Proof: i) We have to check that the $n$-point functions (3.18) satisfy the conditions of Lemma 3.1. Using the product formula (3.18), it is straightforward to verify that conditions i)-iii) hold for all $n \geq 2$. For $i v$ ), we note that $\overline{\rho_{2}(-q,-p)}=\rho_{2}(p, q)$ by the ${ }^{*}$-invariance of $\rho_{2}$, and compute

$$
\overline{\rho_{n}\left(-p_{n}, \ldots,-p_{1}\right)}=\prod_{1 \leq l<r \leq n} \overline{\rho_{2}\left(-p_{r},-p_{l}\right)}=\prod_{1 \leq l<r \leq n} \rho_{2}\left(p_{l}, p_{r}\right)=\rho_{n}\left(p_{1}, \ldots, p_{n}\right) .
$$

Hence $i v$ ) holds, and by definition (3.18), also $v$ ) is satisfied. Thus $\rho \in \mathcal{R}_{0}$.

ii) Here we just have to insert the definition of $\rho_{n}$ (3.18) into (3.9). Let $n \in \mathbb{N}_{0}$ and $k \in\{0, \ldots, n\}$. Then

$$
\begin{aligned}
\frac{\rho_{k}\left(p_{1}, \ldots, p_{k}\right) \rho_{n-k}\left(p_{k+1}, \ldots, p_{n}\right)}{\rho_{n}\left(p_{1}, \ldots, p_{n}\right)} & =\frac{\prod_{1 \leq l^{\prime}<r^{\prime} \leq k} \rho_{2}\left(p_{l^{\prime}}, p_{r^{\prime}}\right) \cdot \prod_{k+1 \leq l^{\prime \prime}<r^{\prime \prime} \leq n} \rho_{2}\left(p_{l^{\prime \prime}}, p_{r^{\prime \prime}}\right)}{\prod_{1 \leq l<r \leq n} \rho_{2}\left(p_{l}, p_{r}\right)} \\
& =\prod_{l=1}^{k} \prod_{r=k+1}^{n} \rho_{2}\left(p_{l}, p_{r}\right)^{-1}
\end{aligned}
$$


and (3.19) follows.

iii) For $\rho$ to be compatible with all quasi-free translationally invariant states, we will show that (3.21) equals 1 for even $n=2 N, k \in\{0, \ldots, 2 N\}$, and $\boldsymbol{p} \in-S_{\mathrm{qf}}^{2 N}$ (3.15). Fixing such $N, k$, let $(\boldsymbol{l}, \boldsymbol{r})=\left\{\left(l_{1}, r_{1}\right), \ldots,\left(l_{N}, r_{N}\right)\right\}$ be a partition of $\{1, \ldots, 2 N\}$ into pairs $\left(l_{j}, r_{j}\right)$ as in (3.15), and $\boldsymbol{p} \in \mathbb{R}^{2 N d}$ with $p_{l_{j}}+p_{r_{j}}=0, j=1, \ldots, N$. We split the partition into three parts: First, the pairs $\left(l_{j}, r_{j}\right)$ with $l_{j}, r_{j} \leq k$, denoted $\left\{\left(\hat{l}_{1}, \hat{r}_{1}\right), \ldots\left(\hat{l}_{L}, \hat{r}_{L}\right)\right\}$, second, the pairs $\left(l_{j}, r_{j}\right)$ with $l_{j} \leq k<r_{j}$, denoted $\left\{\left(\tilde{l}_{1}, \tilde{r}_{1}\right), \ldots,\left(\tilde{l}_{M}, \tilde{r}_{M}\right)\right\}$, and third, the pairs $\left(l_{j}, r_{j}\right)$ with $k<l_{j}, r_{j}$, denoted $\left\{\left(\check{l}_{1}, \check{r}_{1}\right), \ldots,\left(\check{l}_{R}, \check{r}_{R}\right)\right\}$. Clearly, these sets are disjoint, and their union is $\left\{\left(l_{1}, r_{1}\right), \ldots,\left(l_{N}, r_{N}\right)\right\}$, i.e. in particular $\left\{\hat{l}_{1}, \ldots, \hat{l}_{L}, \hat{r}_{1}, \ldots, \hat{r}_{L}, \tilde{l}_{1}, \ldots, \tilde{l}_{M}\right\}=\{1, \ldots, k\}$ and $\left\{\tilde{r}_{1}, \ldots, \tilde{r}_{M}, \check{l}_{1}, \ldots, \breve{l}_{R}, \check{r}_{1}, \ldots, \check{r}_{R}\right\}=$ $\{k+1, \ldots, 2 N\}$.

We now rewrite (3.21) using this splitting as well as the support condition $p_{l_{j}}+p_{r_{j}}=0$ and the properties (3.17), which give

$$
\begin{aligned}
\prod_{l=1}^{k} \prod_{r=k+1}^{2 N} \rho_{2}\left(p_{l}, p_{r}\right)^{-1} & =\prod_{r=k+1}^{2 N}\left(\prod_{i=1}^{L}\left(\rho_{2}\left(p_{\hat{l}_{i}}, p_{r}\right)^{-1} \rho_{2}\left(p_{\hat{r}_{i}}, p_{r}\right)^{-1}\right) \cdot \prod_{j=1}^{M} \rho_{2}\left(p_{\tilde{l}_{j}}, p_{r}\right)^{-1}\right) \\
& =\prod_{r=k+1}^{2 N}\left(\prod_{i=1}^{L}\left(\rho_{2}\left(p_{\hat{l}_{i}}, p_{r}\right)^{-1} \rho_{2}\left(-p_{\hat{l}_{i}}, p_{r}\right)^{-1}\right) \cdot \prod_{j=1}^{M} \rho_{2}\left(p_{\tilde{l}_{j}}, p_{r}\right)^{-1}\right) \\
& =\prod_{j=1}^{M} \prod_{r=k+1}^{2 N} \rho_{2}\left(p_{\tilde{l}_{j}}, p_{r}\right)^{-1} \\
& =\prod_{j=1}^{M}\left(\prod_{t=1}^{R}\left(\rho_{2}\left(p_{\tilde{l}_{j}}, p_{\check{l}_{t}}\right)^{-1} \rho_{2}\left(p_{\tilde{l}_{j}}, p_{\check{r}_{t}}\right)^{-1}\right) \prod_{i=1}^{M} \rho_{2}\left(p_{\tilde{l}_{j}}, p_{\tilde{r}_{i}}\right)^{-1}\right) \\
& =\prod_{j=1}^{M}\left(\prod_{t=1}^{R}\left(\rho_{2}\left(p_{\tilde{l}_{j}}, p_{\check{l}_{t}}\right)^{-1} \rho_{2}\left(p_{\tilde{l}_{j}},-p_{\breve{l}_{t}}\right)^{-1}\right) \prod_{i=1}^{M} \rho_{2}\left(p_{\tilde{l}_{j}}, p_{\tilde{r}_{i}}\right)^{-1}\right) \\
& =\prod_{j=1}^{M} \prod_{i=1}^{M} \rho_{2}\left(p_{\tilde{l}_{j}}, p_{\tilde{l}_{i}}\right) .
\end{aligned}
$$

Using the *-invariance of $\rho_{2}$, and (3.17), we get $\rho_{2}(p, p)={\overline{\rho_{2}(-p,-p)}}^{*}{\overline{\rho_{2}(-p, p)}}^{-1}=1$. Hence in the product $\prod_{i, j}^{M} \rho_{2}\left(p_{\tilde{l}_{j}}, p_{\tilde{l}_{i}}\right)$ the diagonal terms $\rho_{2}\left(p_{\tilde{l}_{j}}, p_{\tilde{l}_{j}}\right)=1$ drop out. The off-diagonal terms appear in reciprocal pairs $\rho_{2}\left(p_{\tilde{l}_{i}}, p_{\tilde{l}_{j}}\right)$ and $\rho_{2}\left(p_{\tilde{l}_{j}}, p_{\tilde{l}_{i}}\right)=\rho_{2}\left(p_{\tilde{l}_{i}}, p_{\tilde{l}_{j}}\right)^{-1}$, and therefore drop out as well. As the partition $(\boldsymbol{l}, \boldsymbol{r})$ was arbitrary, the compatibility of $\rho$ and $\omega$ follows.

Replacing $\rho_{2}$ by $1 / \rho_{2}$, we also have compatibility of $\rho^{-1}$ and $\omega$. The equation $\omega\left(f_{n, k, \pm}\right)=$ $\omega\left(f_{n}\right)$ is just a reformulation of these compatibility statements.

iv) As $\rho_{2}$ satisfies (3.17), and is invariant under the ${ }^{*}$-operation (3.2), we have

$$
\overline{\rho_{2}(p, q)}=\rho_{2}(-q,-p)=\rho_{2}(q, p)=\rho_{2}(p, q)^{-1} .
$$

In view of the product form of the $\rho_{n}$, this implies $\overline{\rho_{n}}=1 / \rho_{n}$. But the $n$-point functions of the opposite deformation $\rho^{\prime}$ are the conjugates of the $\rho_{n}$ (3.8). Hence $\rho^{\prime}=\rho^{-1}$.

Having reduced the problem of finding deformations compatible with quasi-free translationally invariant states to conditions on the two-point function $\rho_{2}$, we next solve these conditions by discussing suitable two-point functions. These will be realized in terms of a deformation function $R$ and an admissible matrix $Q$, defined below. 
Definition 3.4 A deformation function is a smooth function $R: \mathbb{R} \rightarrow \mathbb{C}$ such that

i)

$$
R(a)^{-1}=\overline{R(a)}=R(-a), \quad R(0)=1
$$

ii) For each $k \in \mathbb{N}$, there exists $C_{k}, N_{k}>0$, such that

$$
\left|\frac{\partial^{k} R(a)}{\partial a^{k}}\right| \leq C_{k}\left(1+a^{2}\right)^{N_{k}}, \quad a \in \mathbb{R}
$$

iii) The Fourier transform $\tilde{R} \in \mathscr{S}_{1}^{\prime}$ of $R$ has support on the positive half line.

Note that the support restriction on $\tilde{R}$ amounts to requiring that $R$ has an analytic continuation to the upper half plane. More precisely [RS75, Thm. IX.16], any deformation function is the boundary value in the sense of $\mathscr{S}_{1}^{\prime}$ of a function analytic in the upper half plane, satisfying polynomial bounds at infinity and at the real boundary. Conversely, if $R$ is a function analytic on the upper half plane, satisfying suitable polynomial bounds, then its distributional boundary value on the real line exists, and its Fourier transform has support in the right half line. As concrete examples, consider the functions

$$
R(a)=e^{i c a} \prod_{k=1}^{N} \frac{z_{k}-a}{z_{k}+a}, \quad c \geq 0, \operatorname{Im} z_{1}, \ldots, \operatorname{Im} z_{N} \geq 0,
$$

where with each $z_{k}$, also $-\overline{z_{k}}$ is contained in the set of zeros $\left\{z_{1}, \ldots, z_{N}\right\}$. As these functions satisfy the first two conditions of Definition 3.4 and furthermore have bounded analytic continuations to the upper half plane, they are examples of deformation functions.

Lemma 3.5 Let $R$ be a deformation function, and let $Q$ be $a(d \times d)$-matrix which is antisymmetric w.r.t. the Minkowski inner product on $\mathbb{R}^{d}$, and satisfies

$$
\Lambda Q \Lambda^{-1}=\left\{\begin{aligned}
Q & ; \quad \Lambda \in \mathcal{L}_{+}^{\uparrow} \text { with } \Lambda W_{0}=W_{0} \\
-Q & ; \quad \Lambda \in \mathcal{L}_{+}^{\downarrow} \text { with } \Lambda W_{0}=W_{0}
\end{aligned}\right.
$$

Then the deformation two-point function

$$
\rho_{2}(p, q):=R(-p \cdot Q q)
$$

satisfies all assumptions of Proposition 3.3.

Proof: Checking the conditions i) $-i v$ ) of Lemma 3.1, and the additional properties (3.17) is a matter of straightforward computation making use of the listed properties of $R$, and the antisymmetry and partial Lorentz invariance of $Q$. Note that the minus sign for time-reversing Lorentz transformations which appears in (3.25) cancels against the complex conjugation of $\alpha_{\Lambda}, \Lambda \in \mathcal{L}_{+}^{\downarrow}$, since $\overline{R(-a)}=R(a)$.

Given a deformation map $\rho=\rho(R, Q)$ of the form described above, it is straightforward to check that a general Poincaré transformation $(x, \Lambda) \in \mathcal{P}_{+}$acts on the associated deformed product according to

$$
\alpha_{x, \Lambda}\left(f \otimes_{\rho(R, Q)} g\right)=\alpha_{x, \Lambda}(f) \otimes_{\rho\left(R, \pm \Lambda Q \Lambda^{-1}\right)} \alpha_{x, \Lambda}(g), \quad f, g \in \underline{\mathscr{S}},
$$


where the \pm -sign is "+" for orthochronous and "_" for non-orthochronous $\Lambda$. This identity can easily be verified on the basis of (3.19) and (3.22).

It has been shown in [GL07 that the most general matrix satisfying (3.25) is, in case the spacetime dimension is $d=4$ or $d \neq 4$,

$$
Q=\left(\begin{array}{cccc}
0 & \kappa & 0 & 0 \\
\kappa & 0 & 0 & 0 \\
0 & 0 & 0 & \kappa^{\prime} \\
0 & 0 & -\kappa^{\prime} & 0
\end{array}\right), \quad Q=\left(\begin{array}{ccccc}
0 & \kappa & 0 & \cdots & 0 \\
\kappa & 0 & 0 & \cdots & 0 \\
0 & 0 & 0 & \cdots & 0 \\
\vdots & \vdots & \vdots & \ddots & \vdots \\
0 & 0 & 0 & \cdots & 0
\end{array}\right),
$$

with arbitrary parameters $\kappa, \kappa^{\prime} \in \mathbb{R}$. For Lorentz transformations $\Lambda$ which map the wedge $W_{0}$ onto its causal complement $W_{0}^{\prime}$, one has

$$
\Lambda Q \Lambda^{-1}=\left\{\begin{array}{rl}
-Q & ; \quad \Lambda \in \mathcal{L}_{+}^{\uparrow} \text { with } \Lambda W_{0}=W_{0}^{\prime} \\
Q \quad ; & \Lambda \in \mathcal{L}_{+}^{\downarrow} \text { with } \Lambda W_{0}=W_{0}^{\prime}
\end{array} .\right.
$$

This implies that for fixed $R$, the opposite deformation is given by inverting the $\operatorname{sign}$ of $Q$,

$$
\alpha_{j}\left(f \otimes_{\rho(R, Q)} g\right)=\alpha_{j}(f) \otimes_{\rho(R,-Q)} \alpha_{j}(g), \quad f, g \in \underline{\mathscr{S}} .
$$

We also mention that the deformations $\rho(R, Q)$ naturally lead to one-parameter families of deformation maps $\rho(R, \lambda \cdot Q), \lambda \in \mathbb{R}$. In the limit $\lambda \rightarrow 0$, we recover the undeformed product.

Proposition 3.6 Let $R$ be a deformation function and $Q$ a real $(d \times d)$-matrix. Then, for all $f, g \in \underline{\mathscr{S}}$

$$
\lim _{\lambda \rightarrow 0} f \otimes_{\rho(R, \lambda \cdot Q)} g=f \otimes g
$$

Proof: As $R(0)=1$, the functions $r_{\lambda}\left(p_{1}, \ldots, p_{n}\right):=\prod_{l, r} R\left(\lambda p_{l} \cdot Q p_{r}\right)$ (3.19) appearing in the product $\otimes_{\rho(R, \lambda \cdot Q)}$ converge pointwise to the constant function 1 as $\lambda \rightarrow 0$. This limit is also valid in a stronger topology: Making use of the polynomial boundedness of the derivatives of $R$, it is not difficult to show that for any multi index $\boldsymbol{\mu} \in \mathbb{N}^{n d}$, there exists $N(\boldsymbol{\mu}) \in \mathbb{R}$ such that

$$
\lim _{\lambda \rightarrow 0} \sup _{\boldsymbol{p} \in \mathbb{R}^{n d}} \frac{\left|\partial_{\boldsymbol{p}}^{\boldsymbol{\mu}}\left(r_{\lambda}(\boldsymbol{p})-1\right)\right|}{\left(1+\|\boldsymbol{p}\|^{2}\right)^{N(\boldsymbol{\mu})}}=0
$$

It then follows by straightforward estimates that $\left(f \otimes_{\rho(R, \lambda \cdot Q)} g\right)_{n}=r_{\lambda} \cdot(f \otimes g)_{n} \rightarrow(f \otimes g)_{n}$ as $\lambda \rightarrow 0$, in the topology of $\mathscr{S}_{n}, n \in \mathbb{N}$. This implies the claimed limit (3.31).

The simplest non-trivial deformation function is $R(a):=e^{i a}$. This example was studied in GL08. For this function, the corresponding deformed product

$$
\left.\widetilde{\left(f \otimes_{\rho} g\right.}\right)_{n}\left(p_{1}, \ldots, p_{n}\right)=\sum_{k=0}^{n} e^{i\left(p_{1}+\ldots+p_{k}\right) \cdot Q\left(p_{k+1}+\ldots+p_{n}\right)} \tilde{f}_{k}\left(p_{1}, \ldots, p_{k}\right) \tilde{g}_{n-k}\left(p_{k+1}, \ldots, p_{n}\right)
$$

can also be written as

$$
\left(f \otimes_{\rho} g\right)_{n}\left(x_{1}, \ldots, x_{n}\right)=(2 \pi)^{-d} \int d q d y e^{-i q \cdot y}\left(\alpha_{Q q} f \otimes \alpha_{y} g\right)_{n}\left(x_{1}, \ldots, x_{n}\right) .
$$


It is thus identical to the Rieffel-deformation [Rie92] of the tensor product $\otimes$ with the $\mathbb{R}^{d}$-action $\left.\alpha\right|_{\mathbb{R}^{d}}$ GL08. In particular, it follows that all translationally invariant states are compatible with this deformation, a fact proven in a $C^{*}$-framework in Rie93. In our present setting, we immediately see that because of the antisymmetry of $Q$, we have $e^{-i\left(p_{1}+\ldots+p_{k}\right) \cdot Q\left(p_{k+1}+\ldots+p_{n}\right)}=1$ for all $\boldsymbol{p} \in S_{\text {inv }}^{n}$ (3.12). By Proposition ??, this implies the compatibility of this deformation with all translationally invariant (not necessarily quasi-free) states.

However, for the deformations given by a general deformation function $R$, the restriction to quasi-free states is necessary. In fact, assume that the $n$-point functions $\rho_{n}$ defined in terms of $R$ by (3.26) and (3.18) satisfy (3.11) on $S_{\text {inv }}^{n}$ (3.12). Taking $n=4$ and $k=1$ in (3.11), we then have $R\left(p_{1} \cdot Q p_{2}\right) R\left(p_{1} \cdot Q p_{3}\right) R\left(p_{1} \cdot Q p_{4}\right)=1$ for all $p$ with $p_{1}+\ldots+p_{4}=0$. Inserting $p_{4}=-\left(p_{1}+p_{2}+p_{3}\right)$ and making use of the antisymmetry of $Q$ as well as (3.22) yields the condition

$$
R\left(p_{1} \cdot Q p_{2}\right) R\left(p_{1} \cdot Q p_{3}\right)=R\left(p_{1} \cdot Q p_{2}+p_{1} \cdot Q p_{3}\right) .
$$

Since $p_{1}, p_{2}, p_{3}$ can be chosen independently, this condition is only satisfied for $R(a)=e^{i c a}$. We will therefore restrict our attention to quasi-free translationally invariant states $\omega$ in the following.

We now consider the question under which conditions a multiplicative deformation $\rho$ is wedgelocal in the GNS representation associated with a quasi-free translationally invariant state $\omega$. As we have been working with the full tensor algebra $\mathscr{\mathscr { L }}$ instead of its quotient $\mathscr{\mathscr { L }} / \mathscr{L}$ by the locality ideal $\mathscr{L}$, we have to consider states annihilating $\mathscr{L}$. Picking such a state $\omega$, we recall from Lemma 2.4 that wedge-locality in the GNS representation of $(\underline{\mathscr{S}}, \omega)$ amounts to

$$
\omega\left(\left(u \otimes_{\rho} f\right) \otimes\left(g^{\prime} \otimes_{\rho^{\prime}} v\right)\right)=\omega\left(\left(u \otimes_{\rho^{\prime}} g^{\prime}\right) \otimes\left(f \otimes_{\rho} v\right)\right)
$$

for all $f \in \underline{\mathscr{S}}\left(W_{0}\right), g^{\prime} \in \alpha_{j}\left(\underline{\mathscr{S}}\left(W_{0}\right)\right)=\underline{\mathscr{S}}\left(W_{0}^{\prime}\right)$, and all $u, v \in \underline{\mathscr{S}}$.

To motivate the following steps, it is instructive to recall the known results about the special case $R(a)=e^{i a}$ first. In this context, (3.32) is known to hold for a translationally invariant state $\omega$ annihilating $\mathscr{L}$ if $\omega$ satisfies also the spectrum condition and $Q$ is admissible in the sense that $Q \overline{V_{+}} \subset \overline{W_{0}}$ [BLS10]. This interplay of locality and spectral properties can be understood as follows. The spectrum condition restricts the supports of the $n$-point functions $\tilde{\omega}_{n}$ to those $\boldsymbol{p} \in \mathbb{R}^{n d}$ with $p_{1}, p_{1}+p_{2}, \ldots, p_{1}+\ldots+p_{n} \in \overline{V_{+}}$(3.13). This implies that in (3.32), we may restrict to $u$ with $\operatorname{supp} \tilde{u} \subset-S_{\text {inv }}^{n}$. For those $u$, in the deformed product

$$
\begin{aligned}
\left(\widetilde{u \otimes_{\rho} f}\right)_{n}\left(p_{1}, \ldots, p_{n}\right) & =\sum_{k=0}^{n} e^{i\left(p_{1}+\ldots+p_{k}\right) \cdot Q\left(p_{k+1}+\ldots+p_{n}\right)} \tilde{u}_{k}\left(p_{1}, \ldots, p_{k}\right) \tilde{f}_{n-k}\left(p_{k+1}, \ldots, p_{n}\right) \\
& =\sum_{k=0}^{n} \tilde{u}_{k}\left(p_{1}, \ldots, p_{k}\right) \alpha_{-Q\left(p_{1}+\ldots+p_{k}\right)}(\tilde{f})_{n-k}\left(p_{k+1}, \ldots, p_{n}\right)
\end{aligned}
$$

only translations of $f$ in the directions $-Q\left(p_{1}+\ldots+p_{k}\right)$ appear, which by admissibility of $Q$ lie in the right wedge $\overline{W_{0}}$. But translations along $x \in \overline{W_{0}}$ preserve the support of $f \in \mathscr{\mathscr { S }}\left(W_{0}\right)$. Similar arguments can be applied to the other terms in (3.32), showing that $g^{\prime} \in \mathscr{\mathscr { L }}\left(W_{0}^{\prime}\right)$ is effectively translated in the opposite direction, so that also the support of $g^{\prime}$ in $W_{0}^{\prime}=-W_{0}$ is preserved. Thus the effect of the deformation consists in shifting the spacelike supports of $f$ and $g^{\prime}$ apart, and the locality condition of $\omega$ then allows to conclude that (3.32) holds GL08.

As we are working here with a family of deformations containing $R(a)=e^{i a}$, we will in the following also require that $\omega$ satisfies the spectrum condition and $Q$ is admissible. This last condition simply amounts to choosing the parameter $\kappa$ appearing in $Q(3.28)$ non-negative. 
The multiplicative deformations given by a function $R$ which are not of exponential form do not simply act as translations on $\mathscr{\mathscr { S }}$, and the preceding locality argument for the case $R(a)=e^{i a}$ has to be adapted. Here the half-sided support of the Fourier transform $\tilde{R}$ (Definition 3.4 iii)) comes into play, which makes it possible to control the effect of the deformed products $\otimes_{\rho}, \otimes_{\rho^{\prime}}$ on the spacetime supports of suitable test functions.

Proposition 3.7 Let $R$ be a deformation function and define, $x \in \mathbb{R}^{d}$,

$$
\begin{aligned}
\tau_{x}^{R}: \underline{\mathscr{S}} \rightarrow \underline{\mathscr{S}}, \\
\widetilde{\left(\tau_{x}^{R} f\right)_{n}}\left(p_{1}, \ldots, p_{n}\right):=\tilde{f}_{n}\left(p_{1}, \ldots, p_{n}\right) \cdot \prod_{k=1}^{n} R\left(x \cdot p_{k}\right) .
\end{aligned}
$$

i.) $\tau_{x}^{R}$ is a continuous automorphism of $\underline{\mathscr{S}}$ for any $x \in \mathbb{R}^{d}$. For $x \in \overline{W_{0}}$, one has

$$
\tau_{ \pm x}^{R}\left(\mathscr{\mathscr { L }}\left( \pm W_{0}\right)\right) \subset \underline{\mathscr{L}}\left( \pm W_{0}\right) .
$$

ii.) Let $n, m \in \mathbb{N}_{0}, h^{ \pm} \in \mathscr{S}_{m}$ with supp $\tilde{h}^{ \pm} \subset \overline{V_{ \pm}}$, and $f \in \mathscr{S}_{n}\left(W_{0}\right), g^{\prime} \in \underline{\mathscr{S}}\left(W_{0}^{\prime}\right)$. Then the deformation map $\rho$ given by $R$ and an admissible matrix $Q$ satisfies

$$
\begin{aligned}
& \operatorname{supp}\left(h^{-} \otimes_{\rho} f\right) \subset \mathbb{R}^{m d} \times\left(W_{0}\right)^{\times n}, \\
& \operatorname{supp}\left(h^{-} \otimes_{\rho^{\prime}} g^{\prime}\right) \subset \mathbb{R}^{m d} \times\left(W_{0}^{\prime}\right)^{\times n}, \\
& \operatorname{supp}\left(g^{\prime} \otimes_{\rho^{\prime}} h^{+}\right) \subset\left(W_{0}^{\prime}\right)^{\times n} \times \mathbb{R}^{m d}, \\
& \operatorname{supp}\left(f \otimes_{\rho} h^{+}\right) \subset\left(W_{0}\right)^{\times n} \times \mathbb{R}^{m d} .
\end{aligned}
$$

Proof: i) The linearity and continuity of each $\tau_{x}^{R}$ is clear. In momentum space, $\tau_{x}^{R}$ multiplies by the tensor product function $R_{x}^{\otimes n}, R_{x}(p):=R(x \cdot p)$. Hence $\tau_{x}^{R}$ is an algebra homomorphism. It is also invertible, with inverse $\left(\tau_{x}^{R}\right)^{-1}=\tau_{-x}^{R}$, because $R(-a)=R(a)^{-1}$. By definition, $\tau_{x}^{R}$ has the identity of $\mathscr{S}$ as a fixed point, and since $\overline{R(-a)}=R(a)$, we also have $\tau_{x}^{R}(f)^{*}=\tau_{x}^{R}\left(f^{*}\right)$, $f \in \underline{\mathscr{S}}$. This shows that $\tau_{x}^{R}$ is an automorphism of $\mathscr{\mathscr { S }}$. Note that although $\tau_{0}^{R}=\mathrm{id}$ and $\left(\tau_{x}^{R}\right)^{-1}=\tau_{-x}^{R}$, the group law $\tau_{x}^{R} \tau_{y}^{R}=\tau_{x+y}^{R}$ holds only if $R$ is of exponential form.

To check the claims about the supports in wedges, let $f \in \underline{\mathscr{L}}\left(W_{0}\right)$, i.e., $\operatorname{supp} f_{n} \subset W_{0}^{\times n}$ for all $n \in \mathbb{N}$. The function $R_{x}^{\otimes n}$ defines a tempered distribution, and therefore has a Fourier transform $\tilde{R}_{x}^{\otimes n}$ in $\mathscr{S}_{n}^{\prime}$ such that $\tau_{x}^{R}\left(f_{n}\right)=(2 \pi)^{-n d / 2} \tilde{R}_{x}^{\otimes n} * f_{n}$. Explicitly, $\boldsymbol{y}, \boldsymbol{z}, \boldsymbol{p} \in \mathbb{R}^{n d}$,

$$
\begin{aligned}
\left(\tau_{x}^{R} f\right)_{n}(\boldsymbol{y}) & =(2 \pi)^{-n d / 2} \int d \boldsymbol{z} f_{n}(\boldsymbol{y}-\boldsymbol{z}) \int d \boldsymbol{p} \prod_{k=1}^{n}\left(e^{-i p_{k} \cdot z_{k}} R\left(x \cdot p_{k}\right)\right) \\
& =(2 \pi)^{-n d / 2} \int d \boldsymbol{z} f_{n}(\boldsymbol{y}-\boldsymbol{z}) \int d \boldsymbol{p} \prod_{k=1}^{n}\left(e^{-i p_{k} \cdot z_{k}}(2 \pi)^{-1 / 2} \int d \lambda_{k} e^{i \lambda_{k}\left(x \cdot p_{k}\right)} \tilde{R}\left(\lambda_{k}\right)\right) \\
& =(2 \pi)^{+n(d-1) / 2} \int d \lambda_{1} \cdots d \lambda_{n} \tilde{R}\left(\lambda_{1}\right) \cdots \tilde{R}\left(\lambda_{n}\right) \int d \boldsymbol{z} f_{n}(\boldsymbol{y}-\boldsymbol{z}) \prod_{k=1}^{n} \delta\left(z_{k}-\lambda_{k} x\right) \\
& =(2 \pi)^{+n(d-1) / 2} \int d \lambda_{1} \cdots d \lambda_{n} \tilde{R}\left(\lambda_{1}\right) \cdots \tilde{R}\left(\lambda_{n}\right) f_{n}\left(y_{1}-\lambda_{1} x, \ldots, y_{n}-\lambda_{n} x\right) .
\end{aligned}
$$

The wedge $W_{0}$ has the two geometric properties $\lambda W_{0} \subset \overline{W_{0}}$ for $\lambda \geq 0$ and $W_{0}+\overline{W_{0}} \subset W_{0}$. Since $\operatorname{supp} \tilde{R} \subset \mathbb{R}_{+}$, all $\lambda_{k}$ appearing in this integral are positive, and since $x \in \overline{W_{0}}$, we have $\lambda_{1} x, \ldots, \lambda_{n} x \in \overline{W_{0}}$. Taking into account that the support of $f_{n}$ lies in $W_{0}^{\times n}$, we find 
$\operatorname{supp}\left(\tau_{x}^{R} f\right)_{n} \subset \operatorname{supp} f_{n}+{\overline{W_{0}}}^{\times n} \subset W_{0}^{\times n}+{\overline{W_{0}}}^{\times n} \subset W_{0}^{\times n}$, and hence $\tau_{x}^{R} \underline{\mathscr{S}}\left(W_{0}\right) \subset \underline{\mathscr{S}}\left(W_{0}\right)$. The arguments leading to $\tau_{-x}^{R}\left(\underline{\mathscr{S}}\left(-W_{0}\right)\right) \subset \mathscr{S}\left(-W_{0}\right)$ are completely analogous.

ii) The deformed product $h^{-} \otimes_{\rho} f$ can be expressed with the shift automorphisms $\tau_{x}^{R}$ (3.33) as, $\boldsymbol{k} \in \mathbb{R}^{m d}, \boldsymbol{p} \in \mathbb{R}^{\text {nd }}$,

$$
\begin{aligned}
\left(h^{-} \otimes_{\rho} f\right)^{\sim}(\boldsymbol{k}, \boldsymbol{p}) & =\tilde{h}^{-}(\boldsymbol{k}) \prod_{l=1}^{m} \prod_{r=1}^{n} R\left(k_{l} \cdot Q p_{r}\right) \cdot \tilde{f}(\boldsymbol{p}) \\
& =\tilde{h}^{-}(\boldsymbol{k})\left(\tau_{-Q k_{1}}^{R} \cdots \tau_{-Q k_{m}}^{R} f\right)^{\sim}(\boldsymbol{p}) .
\end{aligned}
$$

As supp $\tilde{h}^{-} \subset \overline{V_{-}}$and $Q$ is admissible, the vectors $-Q k_{1}, \ldots,-Q k_{n}$ all lie in $\overline{W_{0}}$, and by part a), we have $\tau_{-Q k_{1}}^{R} \cdots \tau_{-Q k_{m}}^{R} f \in \mathscr{S}_{n}\left(W_{0}\right)$. Hence $h^{-} \otimes_{\rho} f$ has support in $\mathbb{R}^{m d} \times W_{0}^{\times n}$.

In comparison, in $\left(g^{\prime} \otimes_{\rho^{\prime}} h^{+}\right)$, the support of $g^{\prime}$ lies in $-W_{0}$ instead of $W_{0}$, and $\rho$ is replaced by $\rho^{\prime}$. But the opposite deformation is given by the same deformation function $R$, and matrix $-Q$ instead of $Q$. Hence we can repeat the above argument with shifts $+Q k_{1}, \ldots,+Q k_{m} \in-\overline{W_{0}}$, preserving the support of $g^{\prime}$ in $-W_{0}$, i.e. $\operatorname{supp}\left(g^{\prime} \otimes_{\rho^{\prime}} h^{+}\right) \subset\left(W_{0}^{\prime}\right)^{\times n} \times \mathbb{R}^{m d}$.

The third and fourth function can be rewritten as

$$
\left(g^{\prime} \otimes_{\rho^{\prime}} h^{+}\right)=\left(\left(h^{+}\right)^{*} \otimes_{\rho^{\prime}}\left(g^{\prime}\right)^{*}\right)^{*}, \quad\left(f \otimes_{\rho} h^{+}\right)=\left(\left(h^{+}\right)^{*} \otimes_{\rho} f^{*}\right)^{*} .
$$

As the *-involution preserves supports in spacetime, but reflects supports in momentum space about the origin, we have $\operatorname{supp}\left(\tilde{h}^{+}\right)^{*} \subset \overline{V_{-}}$, supp $\left(g^{\prime}\right)^{*} \subset\left(-W_{0}\right)$, supp $f^{*} \subset W_{0}$, as in the first two functions $h^{-} \otimes_{\rho} f, h^{-} \otimes_{\rho^{\prime}} g^{\prime}$. Taking also into account $\left(\mathscr{S}_{m} \otimes \mathscr{S}_{n}\left( \pm W_{0}\right)\right)^{*}=\mathscr{S}_{n}\left( \pm W_{0}\right) \otimes \mathscr{S}_{m}$, the claim about the supports of $\left(g^{\prime} \otimes_{\rho^{\prime}} h^{+}\right)$and $\left(f \otimes_{\rho} h^{+}\right)$follows.

As a preparation for the wedge-locality proof, we recall two facts about quasi-free states satisfying the spectrum condition respectively states vanishing on the locality ideal.

Lemma 3.8 Let $\omega$ be a quasi-free translationally invariant state on $\mathscr{L}$ which satisfies the spectrum condition, and consider some $f \in \underline{\mathscr{S}}$ and the vector $\Psi_{\omega}(f)$ representing $f$ in the GNS representation space of $(\underline{\mathscr{S}}, \omega)$. If

$$
\omega\left(h^{-} \otimes f\right)=0 \quad \text { for all } h^{-} \in \underline{\mathscr{S}} \text { with supp } \tilde{h}^{-} \subset \overline{V_{-}},
$$

then $\Psi_{\omega}(f)=0$.

Proof: By the GNS construction, we have $\omega\left(h^{-} \otimes f\right)=\left\langle\Psi_{\omega}\left(\left(h^{-}\right)^{*}\right), \Psi_{\omega}(f)\right\rangle$, where the momentum space support of $\left(h^{-}\right)^{*}$ is $\operatorname{supp}\left(\tilde{h}^{-}\right)^{*} \subset \overline{V^{+}}$. We thus have to show that the space $\mathcal{D}_{+} \subset \mathcal{H}_{\omega}$ of all $\Psi_{\omega}\left(h^{+}\right)$, where $\operatorname{supp} \tilde{h}^{+} \subset \overline{V_{+}}$, is dense. This is a consequence of $\omega$ being quasi-free and satisfying the spectrum condition. In fact, in this situation, $\omega_{2}$ has the form (3.16) with a measure $w$ on $\overline{V_{+}}$, and the GNS representation space $\mathcal{H}_{\omega}$ is the Bose Fock space over the single particle space $L^{2}\left(\overline{V_{+}}, w(p) d p\right)$. For functions $h_{n}^{+} \in \mathscr{S}_{n}$ whose support in momentum space does not intersect the backward lightcone, $\Psi_{\omega}\left(h_{n}^{+}\right)$is a vector in the $n$-particle space $L^{2}\left(\overline{V_{+}}, w(p) d p\right)^{\otimes_{\mathrm{sym}} n}$, given by symmetrization in all variables of the Fourier transform $\tilde{h}_{n}^{+}$. The $n$-particle vectors obtained in this manner form a dense subspace of the $n$-particle space. Since we can take arbitrary $n$, the density of $\mathcal{D}_{+}$follows. 
Lemma 3.9 Let $F \in \mathscr{S}_{n+m}, G \in \mathscr{S}_{n^{\prime}+m^{\prime}}, n, m, n^{\prime}, m^{\prime} \in \mathbb{N}_{0}$, such that supp $F \subset \mathbb{R}^{m d} \times$ $\left(W_{0}\right)^{\times n}$ and supp $G \subset\left(W_{0}^{\prime}\right)^{\times n^{\prime}} \times \mathbb{R}^{m^{\prime} d}$. Let $\tau: \mathscr{S}_{m+n+n^{\prime}+m^{\prime}} \rightarrow \mathscr{S}_{m+n+n^{\prime}+m^{\prime}}$ denote the flip

$$
(\tau H)\left(\boldsymbol{y}, \boldsymbol{x}, \boldsymbol{x}^{\prime}, \boldsymbol{y}^{\prime}\right):=H\left(\boldsymbol{y}, \boldsymbol{x}^{\prime}, \boldsymbol{x}, \boldsymbol{y}^{\prime}\right), \quad \boldsymbol{y} \in \mathbb{R}^{m d}, \boldsymbol{x} \in \mathbb{R}^{n d}, \boldsymbol{x}^{\prime} \in \mathbb{R}^{n^{\prime} d}, \boldsymbol{y}^{\prime} \in \mathbb{R}^{m^{\prime} d} .
$$

Then for each state $\omega$ on $\mathscr{S}$ which annihilates the locality ideal, we have

$$
\omega(F \otimes G)=\omega(\tau(F \otimes G)) .
$$

Proof: In view of the support properties of $F$ and $G$, we can represent these functions as $F=\sum_{t=1}^{\infty} l^{(t)} \otimes r^{(t)}, G=\sum_{t=1}^{\infty} a^{(t)} \otimes b^{(t)}$, with $l^{(t)} \in \mathscr{S}_{m}, r^{(t)} \in \mathscr{S}_{n}\left(W_{0}\right), a^{(t)} \in \mathscr{S}_{n^{\prime}}\left(W_{0}^{\prime}\right)$, $b^{(t)} \in \mathscr{S}_{m^{\prime}}$, and these series converge in the topology of $\mathscr{\mathscr { S }}$. Because the supports of the $r^{(t)}$ and $a^{(t)}$ are spacelike separated and $\omega$ annihilates the locality ideal, we have

$$
\begin{aligned}
\sum_{t=1}^{T} \sum_{s=1}^{S} \omega\left(l^{(t)} \otimes r^{(t)} \otimes a^{(s)} \otimes b^{(s)}\right) & =\sum_{t=1}^{T} \sum_{s=1}^{S} \omega\left(l^{(t)} \otimes a^{(s)} \otimes r^{(t)} \otimes b^{(s)}\right) \\
& =\omega\left(\tau\left(\sum_{t=1}^{T} \sum_{s=1}^{S} l^{(t)} \otimes r^{(t)} \otimes a^{(s)} \otimes b^{(s)}\right)\right) .
\end{aligned}
$$

Making use of the continuity of $\tau$ and $\omega$, the equality (3.40) follows from the above calculation in the limit $T \rightarrow \infty, S \rightarrow \infty$.

Theorem 3.10 Let $R$ be a deformation function, and let $Q$ be an admissible matrix. Then the deformation $\rho$ given by $R$ and $Q$ via (3.26) and (3.18) is wedge-local in any quasi-free translationally invariant state $\omega$ which satisfies the spectrum condition and vanishes on the locality ideal.

Proof: Let $n, n^{\prime} \in \mathbb{N}_{0}$, and $f \in \mathscr{S}_{n}\left(W_{0}\right), g^{\prime} \in \mathscr{S}_{n^{\prime}}\left(W_{0}^{\prime}\right)$. We have to show that $\phi_{\omega}^{\rho}(f)$ and $\phi_{\omega}^{\rho^{\prime}}\left(g^{\prime}\right)$ commute on the Wightman domain $\phi_{\omega}(\underline{\mathscr{S}}) \Omega_{\omega}$ in the GNS space $\mathcal{H}_{\omega}$. In view of Lemma 2.4. this is equivalent to showing that for arbitrary $h^{+} \in \mathscr{S}_{m}, h^{-} \in \mathscr{S}_{m^{\prime}}, m, m^{\prime} \in \mathbb{N}_{0}$, one of the following equivalent equations holds,

$$
\begin{aligned}
\omega\left(h^{-} \otimes\left(f \otimes_{\rho}\left(g^{\prime} \otimes_{\rho^{\prime}} h^{+}\right)\right)\right) & =\omega\left(h^{-} \otimes\left(g^{\prime} \otimes_{\rho^{\prime}}\left(f \otimes_{\rho} h^{+}\right)\right)\right) \\
\Longleftrightarrow \omega\left(\left(h^{-} \otimes_{\rho} f\right) \otimes\left(g^{\prime} \otimes_{\rho^{\prime}} h^{+}\right)\right) & =\omega\left(\left(h^{-} \otimes_{\rho^{\prime}} g^{\prime}\right) \otimes\left(f \otimes_{\rho} h^{+}\right)\right) \\
\Longleftrightarrow \omega\left(\left(\left(h^{-} \otimes_{\rho} f\right) \otimes_{\rho^{\prime}} g^{\prime}\right) \otimes h^{+}\right) & =\omega\left(\left(\left(h^{-} \otimes_{\rho^{\prime}} g^{\prime}\right) \otimes_{\rho} f\right) \otimes h^{+}\right) \\
\Longleftrightarrow \omega\left(\left(h^{+}\right)^{*} \otimes\left(\left(g^{\prime}\right)^{*} \otimes_{\rho^{\prime}}\left(f^{*} \otimes_{\rho}\left(h^{-}\right)^{*}\right)\right)\right) & =\omega\left(\left(h^{+}\right)^{*} \otimes\left(f^{*} \otimes_{\rho}\left(\left(g^{\prime}\right)^{*} \otimes_{\rho^{\prime}}\left(h^{-}\right)^{*}\right)\right)\right) .
\end{aligned}
$$

Considering the first equation, we note that by Lemma 3.8, it is sufficient to consider $h^{-}$with $\operatorname{supp} \tilde{h}^{-} \subset \overline{V_{-}}$. Considering the last equation, we can apply Lemma 3.8 again, and see that we may restrict to $h^{+}$with $\operatorname{supp}\left(\tilde{h}^{+}\right)^{*} \subset \overline{V_{-}}$, or, equivalently, supp $\tilde{h}^{+} \subset \overline{V_{+}}$.

So let $h^{ \pm}$have the specified momentum space supports, and consider the equation in question in the form (3.41). In view of the support properties of $f, g^{\prime}$, we can apply Proposition 3.7. Introducing the abbreviations $F^{-}:=h^{-} \otimes_{\rho} f, F^{+}:=f \otimes_{\rho} h^{+}, G^{-}:=h^{-} \otimes_{\rho^{\prime}} g^{\prime}, G^{+}:=g^{\prime} \otimes_{\rho^{\prime}} h^{+}$, we have

$$
\begin{aligned}
& \operatorname{supp} F^{-} \subset \mathbb{R}^{m d} \times\left(W_{0}\right)^{\times n}, \quad \operatorname{supp} G^{+} \subset\left(W_{0}^{\prime}\right)^{\times n^{\prime}} \times \mathbb{R}^{m^{\prime} d}, \\
& \operatorname{supp} G^{-} \subset \mathbb{R}^{m d} \times\left(W_{0}^{\prime}\right)^{\times n^{\prime}}, \quad \operatorname{supp} F^{+} \subset\left(W_{0}\right)^{\times n} \times \mathbb{R}^{m d} \text {. }
\end{aligned}
$$


Application of Lemma 3.9 now yields $\omega\left(F^{-} \otimes G^{+}\right)=\omega\left(\tau\left(F^{-} \otimes G^{+}\right)\right)$with the flip $\tau$ (3.39). Note that $\tau$ also acts in momentum space by interchanging the two middle variables.

To complete the proof, we now show that $\omega\left(\tau\left(F^{-} \otimes G^{+}\right)\right)=\omega\left(G^{-} \otimes F^{+}\right)$by exploiting the compatibility of $\omega$ with $\rho, \rho^{\prime}$ in the form expressed in (3.20). We can thus multiply $\left(G^{-} \otimes F^{+}\right)^{\sim}$ with various factors of $R( \pm p \cdot Q q)$ without changing its expectation value in $\omega$. Explicitly, $\boldsymbol{k} \in \mathbb{R}^{m}, \boldsymbol{p} \in \mathbb{R}^{n}, \boldsymbol{p}^{\prime} \in \mathbb{R}^{n^{\prime}}, \boldsymbol{k}^{\prime} \in \mathbb{R}^{m^{\prime}}$,

$$
\left(G^{-} \otimes F^{+}\right)^{\sim}\left(\boldsymbol{k}, \boldsymbol{p}^{\prime}, \boldsymbol{p}, \boldsymbol{k}^{\prime}\right)=\tilde{h}^{-}(\boldsymbol{k}) \tilde{g}^{\prime}\left(\boldsymbol{p}^{\prime}\right) \tilde{f}(\boldsymbol{p}) \tilde{h}^{+}\left(\boldsymbol{k}^{\prime}\right) \prod_{l=1}^{m} \prod_{r=1}^{n^{\prime}} R\left(-k_{l} \cdot Q p_{r}^{\prime}\right) \prod_{l=1}^{n} \prod_{r=1}^{m^{\prime}} R\left(p_{l} \cdot Q k_{r}^{\prime}\right),
$$

and we choose once $k=m$ and two point function $\rho_{2}(p, q)=R(-p \cdot Q q)$ in (3.20), and once $k=m+n+n^{\prime}$ and $\rho_{2}(p, q)=R(p \cdot Q q)$. Multiplying $\left(G^{-} \otimes F^{+}\right)^{\sim}$ by these products results in a function $C$, which takes the form

$$
\begin{aligned}
\tilde{C}\left(\boldsymbol{k}, \boldsymbol{p}^{\prime}, \boldsymbol{p}, \boldsymbol{k}^{\prime}\right) & :=\left(G^{-} \otimes F^{+}\right) \sim\left(\boldsymbol{k}, \boldsymbol{p}^{\prime}, \boldsymbol{p}, \boldsymbol{k}^{\prime}\right) \cdot \prod_{l=1}^{m}\left\{\prod_{r=1}^{n^{\prime}} R\left(k_{l} Q p_{r}^{\prime}\right) \cdot \prod_{r=1}^{n} R\left(k_{l} Q p_{r}\right) \cdot \prod_{r=1}^{m^{\prime}} R\left(k_{l} Q k_{r}^{\prime}\right)\right\} \times \\
& \times \prod_{r=1}^{m^{\prime}}\left\{\prod_{l=1}^{m} R\left(-k_{l} Q k_{r}^{\prime}\right) \cdot \prod_{l=1}^{n^{\prime}} R\left(-p_{l}^{\prime} Q k_{r}^{\prime}\right) \cdot \prod_{l=1}^{n} R\left(-p_{l} Q k_{r}^{\prime}\right)\right\} \\
& =\tilde{h}^{-}(\boldsymbol{k}) \tilde{f}(\boldsymbol{p}) \tilde{g}^{\prime}\left(\boldsymbol{p}^{\prime}\right) \tilde{h}^{+}\left(\boldsymbol{k}^{\prime}\right) \cdot \prod_{l=1}^{m} \prod_{r=1}^{n} R\left(k_{l} Q p_{r}\right) \cdot \prod_{l=1}^{n^{\prime}} \prod_{r=1}^{m^{\prime}} R\left(-p_{l}^{\prime} Q k_{r}^{\prime}\right) \\
& =\left(\left(h^{-} \otimes_{\rho} f\right) \otimes\left(g^{\prime} \otimes \rho^{\prime} h^{+}\right)\right)^{\sim}\left(\boldsymbol{k}, \boldsymbol{p}, \boldsymbol{p}^{\prime}, \boldsymbol{k}^{\prime}\right) \\
& =\left(F^{-} \otimes G^{+}\right)^{\sim}\left(\boldsymbol{k}, \boldsymbol{p}, \boldsymbol{p}^{\prime}, \boldsymbol{k}^{\prime}\right),
\end{aligned}
$$

that is, $C=\tau\left(F^{-} \otimes G^{+}\right)$. By construction of $C$, we have $\omega(C)=\omega\left(G^{-} \otimes F^{+}\right)$. Thus we arrive at

$$
\omega\left(F^{-} \otimes G^{+}\right)=\omega\left(\tau\left(F^{-} \otimes G^{+}\right)\right)=\omega(C)=\omega\left(G^{-} \otimes F^{+}\right),
$$

establishing (3.41).

\section{Fock space representations}

As shown in the previous section, there exists a large class of multiplicative deformations on $\underline{\mathscr{S}}$ which are compatible with all quasi-free Wightman states, and therefore give rise to wedge-local deformations of generalized free field theories. In this section, we will for simplicity consider the explicit two point function

$$
\tilde{\omega}_{2}(p, q)=\delta(p+q) \varepsilon_{\boldsymbol{p}}^{-1} \delta\left(p^{0}-\varepsilon_{\boldsymbol{p}}\right), \quad \varepsilon_{\boldsymbol{p}}=\sqrt{\boldsymbol{p}^{2}+m^{2}}, \quad p=\left(p^{0}, \boldsymbol{p}\right) \in \mathbb{R}^{d},
$$

with some fixed mass $m>0$, and discuss multiplicative deformations in the corresponding GNS representation. We will use the notation from Section 2, but generally drop the index $\omega$ on $\phi_{\omega}(f), \Psi_{\omega}(f), \mathcal{D}_{\omega}, \mathcal{H}_{\omega}, \Omega_{\omega}, U_{\omega}$, since we are working with a fixed state here.

Recall that without deformation, the GNS representation $(\phi, \mathcal{H}, \Omega)$ of $(\underline{\mathscr{S}}, \omega)$ describes the model theory of a free scalar field of mass $m$. The representation space $\mathcal{H}$ is the Bose Fock 
space over the single particle space $\mathcal{H}_{1}:=L^{2}\left(\mathbb{R}^{d}, d \mu\right)$ with measure $d \mu(p)=\varepsilon_{\boldsymbol{p}}^{-1} \delta\left(p^{0}-\varepsilon_{\boldsymbol{p}}\right) d p$, and the implementing vector $\Omega$ is the Fock vacuum.

As a consequence of the Poincaré invariance properties of $\omega$, there exists an (anti-)unitary representation $U$ of $\mathcal{P}_{+}$on $\mathcal{H}$, which leaves $\Omega$ invariant, satisfies the spectrum condition, and acts according to $U(x, \Lambda) \Psi(f)=\Psi\left(\alpha_{x, \Lambda} f\right), f \in \underline{\mathscr{S}}$. Explicitly, we have, $\Psi \in \mathcal{H}$,

$$
\begin{aligned}
(U(x, \Lambda) \Psi)_{n}\left(p_{1}, \ldots, p_{n}\right) & =e^{i\left(p_{1}+\ldots+p_{n}\right) \cdot x} \Psi_{n}\left(\Lambda^{-1} p_{1}, \ldots, \Lambda^{-1} p_{n}\right), \\
(U(0, j) \Psi)_{n}\left(p_{1}, \ldots, p_{n}\right) & =\overline{\Psi_{n}\left(-j p_{1}, \ldots,-j p_{n}\right)} .
\end{aligned}
$$

In the following, we will consider a fixed multiplicative deformation $\rho \in \mathcal{R}_{0}$, given by a deformation function $R$ and an admissible matrix $Q$. To keep track of both these objects, we will denote the fields representing $\left(\underline{\mathscr{S}}, \otimes_{\rho}\right)$ as $\phi_{R, Q}(f)$ instead of $\phi^{\rho}(f)$.

Proposition 4.1 Let $R$ be a deformation function, $Q$ an admissible matrix, and $f, g \in \underline{\mathscr{L}}$, $\Psi \in \mathcal{D}$.

i) $\phi_{R, Q}(f)$ is a closable operator containing $\mathcal{D}$ in its domain for any $f \in \underline{\mathscr{S}}$, and the map $\underline{\mathscr{S}} \ni f \mapsto \phi_{R, Q}(f) \Psi \in \mathcal{H}$ is linear and continuous for any $\Psi \in \mathcal{D}$.

ii) For $f, g \in \underline{\mathscr{S}}$,

$$
\begin{aligned}
\phi_{R, Q}(f) \Psi(g) & =\Psi\left(f \otimes_{\rho(R, Q)} g\right), \\
\phi_{R, Q}(f) \phi_{R, Q}(g) & =\phi_{R, Q}\left(f \otimes_{\rho(R, Q)} g\right), \\
\phi_{R, Q}(f)^{*} & \supset \phi_{R, Q}\left(f^{*}\right), \\
\phi_{R, Q}(f) \Omega & =\phi(f) \Omega .
\end{aligned}
$$

iii) Covariance: For $(x, \Lambda) \in \mathcal{P}_{+}^{\uparrow}$, we have

$$
U(x, \Lambda) \phi_{R, Q}(f) U(x, \Lambda)^{-1}=\phi_{R, \Lambda Q \Lambda^{-1}}\left(\alpha_{x, \Lambda} f\right),
$$

and the reflection at the edge of $W_{0}$ acts according to

$$
U(0, j) \phi_{R, Q}(f) U(0, j)=\phi_{R,-Q}\left(\alpha_{j} f\right) .
$$

iv) Wedge-Locality: Let $f \in \underline{\mathscr{L}}\left(W_{0}+a\right), g \in \underline{\mathscr{S}}\left(W_{0}^{\prime}+a\right)$ for some $a \in \mathbb{R}^{d}$. Then

$$
\left[\phi_{R, Q}(f), \phi_{R,-Q}(g)\right] \Psi=0 .
$$

v) Reeh-Schlieder property: For any open set $O \subset \mathbb{R}^{d}$, the subspace

$$
\mathcal{D}_{R, Q}(O):=\phi_{R, Q}(\underline{\mathscr{S}}(O)) \Omega
$$

is dense in $\mathcal{H}$.

vi) $\phi_{R, Q}$ is a weak solution of the Klein-Gordon equation: For $f_{1} \in C_{0}^{\infty}\left(\mathbb{R}^{d}\right)$,

$$
\phi_{R, Q}\left(\left(\square+m^{2}\right) f_{1}\right)=0 .
$$


Proof: The statements in [ii) follow directly from Proposition 2.3 because $\rho$ is compatible with $\omega$, and $\phi_{R, Q}$ is a ${ }^{*}$-representation of $\left(\mathscr{S}, \otimes_{\rho}\right)$. 国) Clearly, each $\phi_{R, Q}(f)$ is defined on the dense domain $\mathcal{D}$, and in view of (4.5) closable. For $g \in \underline{\mathscr{S}}$, the map $f \mapsto \phi_{R, Q}(f) \Psi(g)=$ $\Psi\left(\rho^{-1}(\rho(f) \otimes \rho(g))\right)$ is linear and continuous because $\rho, \rho^{-1}: \underline{\mathscr{S}} \rightarrow \underline{\mathscr{S}}$ and $\Psi: \underline{\mathscr{S}} \rightarrow \mathcal{H}$ are linear and continuous.

The covariance statements in iiii) follow from $U(x, \Lambda) \Psi(f)=\Psi\left(\alpha_{x, \Lambda} f\right), U(0, j) \Psi(f)=$ $\Psi\left(\alpha_{j} f\right)$ and (3.27). iv) In view of the translation covariance iii), it is sufficient to show (4.9) for $a=0$. But this is just a reformulation of Theorem 3.10. The Reeh-Schlieder property $⿴$ ) is known to hold for the undeformed fields, corresponding to $Q=0$. But in view of (4.6), $\mathcal{D}_{R, Q}(O) \supset \mathcal{D}_{0}(O)$, and the density of $\mathcal{D}_{R, Q}(O)$ follows.

The undeformed field $\phi$ is known to be a weak solution of the Klein-Gordon equation. Using (4.6) again, we therefore have $\phi_{R, Q}\left(\left(\square+m^{2}\right) f_{1}\right) \Omega=\phi\left(\left(\square+m^{2}\right) f_{1}\right) \Omega=0$. Now, since $f_{1}$ has compact support, we find $a \in \mathbb{R}^{d}$ such that $W_{0}^{\prime}+a$ lies spacelike to $\operatorname{supp} f_{1}$. For $g \in \underline{\mathscr{L}}\left(W_{0}^{\prime}+a\right)$, we have in view of iv)

$$
\phi_{R, Q}\left(\left(\square+m^{2}\right) f_{1}\right) \phi_{R,-Q}(g) \Omega=\phi_{R,-Q}(g) \phi_{R, Q}\left(\left(\square+m^{2}\right) f_{1}\right) \Omega=0 .
$$

Thus $\phi_{R, Q}\left(\left(\square+m^{2}\right) f_{1}\right)$ vanishes on $\mathcal{D}_{R,-Q}\left(W_{0}^{\prime}+a\right)$. As this subspace is dense by $\left.\llbracket\right)$, we arrive at $\phi_{R, Q}\left(\left(\square+m^{2}\right) f_{1}\right)=0$.

As explained in Section 2, we have now constructed a wedge-local quantum field theory, given by the ${ }^{*}$-algebra $\mathscr{P}_{R}$ generated by all $\phi_{R, Q}(f), f \in \underline{\mathscr{S}}\left(W_{0}\right)$, and

$$
\mathscr{P}_{R}\left(\Lambda W_{0}+x\right):=U(x, \Lambda) \mathscr{P}_{R} U(x, \Lambda)^{-1}, \quad(x, \Lambda) \in \mathcal{P}_{+} .
$$

In view of the transformation property (4.7), the algebra $\mathscr{P}_{R}\left(\Lambda W_{0}+x\right)$ is generated by the field operators $\phi_{R, \pm \Lambda Q \Lambda^{-1}}(f), f \in \mathscr{S}\left(\Lambda W_{0}+x\right)$, where the sign " \pm " refers to orthochronous / antiorthochronous Lorentz transformations. In particular, $\mathscr{P}_{R}\left(W_{0}^{\prime}\right)$ is generated by all $\phi_{R,-Q}(f)$, $f \in \mathscr{S}\left(W_{0}^{\prime}\right)$. Thus the orbit $\mathcal{Q}:=\left\{\Lambda Q \Lambda^{-1}: \Lambda \in \mathcal{L}_{+}\right\}$provides a coordinatization for the different directions of the wedges [GL07, BLS10], whereas the deformation function $R$ labels the kind of deformation used.

It is also possible to proceed from this net of *-algebras of unbounded operators to a corresponding net of von Neumann algebras on $\mathcal{H}$, generated by bounded functions of the fields. However, as we are interested in a field theoretic setting here, we refrain from giving any details.

Before we proceed to studying the observable consequences of the deformation, we point out that with $Q$, also the rescaled matrices $\lambda \cdot Q, \lambda \geq 0$, are admissible. We have thus constructed one-parameter families $\mathscr{P}_{R, \lambda}$ of wedge algebras, representing the deformation maps $\rho(R, \lambda \cdot Q)$. Taking the limit $\lambda \rightarrow 0$ reproduces the undeformed field operators.

Proposition 4.2 Let $R$ be a deformation function and $Q$ an admissible matrix. Then, for any $f \in \underline{\mathscr{S}}, \Psi \in \mathcal{D}$,

$$
\lim _{\lambda \rightarrow 0} \phi_{R, \lambda \cdot Q}(f) \Psi=\phi(f) \Psi
$$

Proof: Since any $\Psi \in \mathcal{D}$ is of the form $\Psi=\Psi(g), g \in \underline{\mathscr{S}}$, we have $\phi_{R, \lambda \cdot Q} \Psi=\Psi\left(f \otimes_{\rho(R, \lambda \cdot Q)} g\right)$. The claim now follows from the continuity of $\Psi: \underline{\mathscr{S}} \rightarrow \mathcal{H}$ and Proposition 3.6 
We now want to compute the deformed field operators $\phi_{R, Q}(f)$ more explicitly in terms of twisted creation and annihilation operators. To this end, we have to introduce some more notation. For $f_{1} \in \mathscr{S}_{1}$, we denote by $f_{1}^{ \pm}(p):=\tilde{f}_{1}( \pm p), p \in H_{m}^{+}$, the restriction of the Fourier transform of $f_{1}$ to the upper and lower mass shell $H_{m}^{ \pm}$. With this notation, $\Psi\left(f_{1}\right)=f_{1}^{+} \in \mathcal{H}_{1}$, and the undeformed field operator has the familiar form

$$
\phi\left(f_{1}\right)=a^{\dagger}\left(\Psi\left(f_{1}\right)\right)+a\left(\Psi\left(f_{1}^{*}\right)\right)=a^{\dagger}\left(f_{1}^{+}\right)+a\left(\overline{f_{1}^{-}}\right), \quad f_{1} \in \mathscr{S}_{1} .
$$

Here $a, a^{\dagger}$ form the standard representation of the canonical commutation relations on $\mathcal{H}$. For $\Psi \in \mathcal{D}, \varphi, \psi \in \mathcal{H}_{1}$,

$$
\begin{aligned}
(a(\varphi) \Psi)_{n}\left(p_{1}, \ldots, p_{n}\right) & :=\sqrt{n+1} \int d \mu(q) \overline{\varphi(q)} \Psi_{n+1}\left(q, p_{1}, \ldots, p_{n}\right), \\
a^{\dagger}(\varphi) & :=a(\varphi)^{*}, \\
{[a(\varphi), a(\psi)] } & =0, \quad\left[a^{\dagger}(\varphi), a^{\dagger}(\psi)\right]=0, \quad\left[a(\varphi), a^{\dagger}(\psi)\right] \Psi=\langle\varphi, \psi\rangle \cdot \Psi .
\end{aligned}
$$

We will also work with the distributional kernels $a^{\#}(p)$ of these operators, related to $a^{\#}(\varphi)$ by $a^{\dagger}(\varphi)=\int d \mu(p) \varphi(p) a^{\dagger}(p)$ and $a(\varphi)=\int d \mu(p) \overline{\varphi(p)} a(p)$, with the commutation relations,

$$
[a(p), a(q)]=0, \quad\left[a^{\dagger}(p), a^{\dagger}(q)\right]=0, \quad\left[a(p), a^{\dagger}(q)\right]=\varepsilon_{\boldsymbol{p}} \delta(p-q) \cdot 1 .
$$

To define deformed versions of these creation/annihilation operators, we introduce the operator-valued function

$$
\begin{aligned}
T_{R}: \mathbb{R}^{d} & \rightarrow \mathcal{B}(\mathcal{H}), \\
\left(T_{R}(x) \Psi\right)_{n}\left(p_{1}, \ldots, p_{n}\right) & :=\prod_{k=1}^{n} R\left(x \cdot p_{k}\right) \Psi_{n}\left(p_{1}, \ldots, p_{n}\right) .
\end{aligned}
$$

It is not difficult to see that quasi-free translationally invariant states are invariant under the shift automorphisms $\tau_{x}^{R}(3.33)$, and the operators $T_{R}(x)$ defined above implement these automorphisms on the GNS space. We will however not need these facts here, and only point out that because of the properties (3.22) of $R$, the operator $T_{R}(x)$ is unitary for any $x \in \mathbb{R}^{d}$, and

$$
T_{R}(x)^{*}=T_{R}(-x)=T_{R}(x)^{-1}, \quad T_{R}(0)=1 .
$$

The operators $T_{R}(x)$ are now used to twist the canonical commutation relations. We define the operator-valued distributions

$$
a_{R, Q}(p):=a(p) T_{R}(Q p), \quad a_{R, Q}^{\dagger}(p)=a^{\dagger}(p) T_{R}(-Q p) .
$$

Making use of the antisymmetry of $Q$ and $R(0)=1$, it is straightforward to check that $a(p)$ and $T_{R}(Q p)$ commute, and thus $a_{R, Q}^{\dagger}(p)=a_{R, Q}(p)^{*}$. Explicitly, the deformed annihilation operator acts as, $\varphi \in \mathcal{H}_{1}, \Psi \in \mathcal{D}$,

$$
\left(a_{R, Q}(\varphi) \Psi\right)_{n}\left(p_{1}, \ldots, p_{n}\right)=\sqrt{n+1} \int d \mu(q) \overline{\varphi(q)} \prod_{k=1}^{n} R\left(Q q \cdot p_{k}\right) \Psi_{n+1}\left(q, p_{1}, \ldots, p_{n}\right),
$$


and $a_{R, Q}^{\dagger}(\varphi)=a_{R, Q}(\varphi)^{*}$. It is instructive to compute the exchange relations of the kernels (4.22) for different matrices $Q, Q^{\prime}$. By straightforward calculation, one gets $a(p) T_{R}(x)=R(x$. $p) \cdot T_{R}(x) a(p)$, and hence

$$
\begin{aligned}
& a_{R, Q}(p) a_{R, Q^{\prime}}\left(p^{\prime}\right)=R\left(p \cdot Q p^{\prime}\right) R\left(p \cdot Q^{\prime} p^{\prime}\right) a_{R, Q^{\prime}}\left(p^{\prime}\right) a_{R, Q}(p) \\
& a_{R, Q}^{\dagger}(p) a_{R, Q^{\prime}}^{\dagger}\left(p^{\prime}\right)=R\left(p \cdot Q p^{\prime}\right) R\left(p \cdot Q^{\prime} p^{\prime}\right) a_{R, Q^{\prime}}^{\dagger}\left(p^{\prime}\right) a_{R, Q}^{\dagger}(p) \\
& a_{R, Q}(p) a_{R, Q^{\prime}}^{\dagger}\left(p^{\prime}\right)=R\left(-p \cdot Q p^{\prime}\right) R\left(-p \cdot Q^{\prime} p^{\prime}\right) a_{R, Q^{\prime}}^{\dagger}\left(p^{\prime}\right) a_{R, Q}(p)+\varepsilon_{\boldsymbol{p}} \delta\left(\boldsymbol{p}-\boldsymbol{p}^{\prime}\right) T_{R}(Q p) T_{R}\left(-Q^{\prime} p\right) .
\end{aligned}
$$

This exchange algebra generalizes the relations of the Moyal-twisted CCR from [GL07]. Putting $Q^{\prime}=Q$, these commutation relations are reminiscent of the Zamolodchikov-Faddeev algebra [ZZ79, Fad84, an observation that will be discussed in Section 6. We also note that for $Q^{\prime}=-Q$, one can use $R(-a)=R(a)^{-1}$ to simplify the above commutators to

$$
\begin{aligned}
& {\left[a_{R, Q}(p), a_{R,-Q}\left(p^{\prime}\right)\right]=0,} \\
& {\left[a_{R, Q}^{\dagger}(p), a_{R,-Q}^{\dagger}\left(p^{\prime}\right)\right]=0,} \\
& {\left[a_{R, Q}(p), a_{R,-Q}^{\dagger}\left(p^{\prime}\right)\right]=\varepsilon_{\boldsymbol{p}} \delta\left(\boldsymbol{p}-\boldsymbol{p}^{\prime}\right) T_{R}(Q p)^{2} .}
\end{aligned}
$$

As $-Q$ corresponds to the reflected wedge $W_{0}^{\prime}$, these exchange relations and the analytic properties of $R$ can also be used for a proof of the wedge-locality in Proposition $4.1 \mathrm{iv}$ ) along the same lines as in Lec03.

Proposition 4.3 The deformed field operators $\phi_{R, Q}\left(f_{1}\right), f_{1} \in \mathscr{S}_{1}$, have the form

$$
\phi_{R, Q}\left(f_{1}\right)=a_{R, Q}^{\dagger}\left(f_{1}^{+}\right)+a_{R, Q}\left(\overline{f_{1}^{-}}\right) .
$$

Proof: Let $f_{1} \in \mathscr{S}_{1}$ with supp $\tilde{f}_{1} \subset V_{-}$, and $g \in \mathscr{S}_{n+1}\left(V_{+}\right), n \in \mathbb{N}_{0}$. Then $\Psi(g) \in \mathcal{H}_{n+1}$, $\Psi\left(f_{1} \otimes_{\rho} g\right) \in \mathcal{H}_{n}$, and for $p_{1}, \ldots, p_{n} \in H_{m}^{+}$, we find, $d \mu(\boldsymbol{p}):=d \mu\left(p_{1}\right) \cdots d \mu\left(p_{n+2}\right)$,

$$
\begin{aligned}
\phi_{Q, R}\left(f_{1}\right) \Psi(g) & =\Psi\left(f_{1} \otimes_{R, Q} g\right) \\
& =\int d \mu(\boldsymbol{p})\left(f_{1} \otimes_{R, Q} g\right)^{\sim}\left(-p_{1}, p_{2}, \ldots, p_{n+2}\right) a\left(p_{1}\right) a^{\dagger}\left(p_{2}\right) \cdots a^{\dagger}\left(p_{n+2}\right) \Omega \\
& =\int d \mu(\boldsymbol{p}) \tilde{f}_{1}\left(-p_{1}\right) \tilde{g}\left(p_{2}, . ., p_{n+2}\right) \prod_{r=2}^{n+2} R\left(-p_{1} \cdot Q p_{r}\right) a\left(p_{1}\right) a^{\dagger}\left(p_{2}\right) \cdots a^{\dagger}\left(p_{n+2}\right) \Omega \\
& =\int d \mu(\boldsymbol{p}) \tilde{f}_{1}\left(-p_{1}\right) \tilde{g}\left(p_{2}, . ., p_{n+2}\right) a\left(p_{1}\right) T_{R}\left(Q p_{1}\right) a^{\dagger}\left(p_{2}\right) \cdots a^{\dagger}\left(p_{n+2}\right) \Omega \\
& =a_{R, Q}\left(\overline{f_{1}^{-}}\right) \Psi(g) .
\end{aligned}
$$

As $g$ and $n$ were arbitrary, we have shown that $\phi_{R, Q}\left(f_{1}\right)$ and $a_{R, Q}\left(\overline{f_{1}^{-}}\right)$coincide on $\mathcal{D}$. Since supp $f_{1}$ does not intersect the upper mass shell, $f_{1}^{+}=0$, and hence the above equation confirms (4.25). Taking adjoints, one also finds, $\Psi \in \mathcal{D}$,

$$
\phi_{R, Q}\left(f_{1}^{*}\right) \Psi=\phi_{R, Q}\left(f_{1}\right)^{*} \Psi=a_{R, Q}\left(\overline{\left(f_{1}^{*}\right)^{-}}\right)^{*} \Psi=a_{R, Q}^{\dagger}\left(\left(f_{1}^{*}\right)^{+}\right) \Psi .
$$

As $\operatorname{supp} \tilde{f}_{1}^{*}=-\operatorname{supp} \tilde{f}_{1} \subset V_{+}$, this implies $\phi_{R, Q}\left(f_{1}\right) \Psi=a_{R, Q}^{\dagger}\left(f_{1}^{+}\right) \Psi$ for all $\Psi \in \mathcal{D}$ and all $f_{1} \in \mathscr{S}_{1}$ with $\operatorname{supp} \tilde{f} \subset V_{+}$. A function $f_{1} \in \mathscr{S}_{1}$ with arbitrary momentum space support can be decomposed according to $f_{1}=g_{1}+h_{1}$ with the support of $\tilde{g}_{1}$ (respectively $\tilde{h}_{1}$ ) not 
intersecting the upper (respectively lower) mass shell. By linearity, this gives (4.25).

It is interesting to note that the deformed field operators can also be expressed as integrals over undeformed fields, similar to the warped convolutions studied in [BLS10]. More precisely, one has, $f_{1} \in \mathscr{S}_{1}$,

$$
\phi_{R, Q}\left(f_{1}\right)=(2 \pi)^{-d} \int d p d x e^{-i p \cdot x} U(x, 1) \phi\left(f_{1}\right) U(-x, 1) T_{R}(-Q p) .
$$

This integral exists as a weak oscillatory integral on vectors $\Psi \in \mathcal{D}$. In fact, for $\operatorname{supp} f_{1} \subset V_{-}$ and $\Psi \in \mathcal{H}$, we obtain, $n \in \mathbb{N}_{0}, q_{1}, \ldots, q_{n} \in H_{m}^{+}$,

$$
\begin{aligned}
(2 \pi)^{-d} \int d p & d x e^{-i p \cdot x}\left(U(x, 1) \phi\left(f_{1}\right) U(-x, 1) T_{R}(-Q p) \Psi\right)_{n}\left(q_{1}, \ldots, q_{n}\right) \\
& =\sqrt{n+1}(2 \pi)^{-d} \int d p d x e^{-i p \cdot x} \int d \mu\left(q_{0}\right) \tilde{f}_{1}\left(-q_{0}\right) e^{-i q_{0} \cdot x}\left(T_{R}(-Q p) \Psi\right)_{n+1}\left(q_{0}, q_{1}, \ldots, q_{n}\right) \\
& =\sqrt{n+1} \int d \mu\left(q_{0}\right) \tilde{f}_{1}\left(-q_{0}\right)\left(T_{R}\left(Q q_{0}\right) \Psi\right)_{n+1}\left(q_{0}, q_{1}, \ldots, q_{n}\right) \\
& =\sqrt{n+1} \int d \mu\left(q_{0}\right) \tilde{f}_{1}\left(-q_{0}\right) \prod_{r=1}^{n} R\left(Q q_{0} \cdot q_{r}\right) \Psi_{n+1}\left(q_{0}, q_{1}, \ldots, q_{n}\right) \\
& =\left(a_{R, Q}\left(\overline{f_{1}^{-}}\right) \Psi\right)_{n}\left(q_{1}, \ldots, q_{n}\right),
\end{aligned}
$$

and an analogous calculation can be carried out for the creation operator, establishing (4.26). However, the integral formula (4.26) reproduces the higher deformed fields $\phi_{R, Q}\left(f_{n}\right), n \geq 2$, only if $R$ is of the exponential form $R(a)=e^{i c a}$. In this case, $T_{R}(x)=U(x, 1)$, and (4.26) coincides with the warped convolution of $\phi(f)$ by the translation representation $\left.U\right|_{\mathbb{R}^{d}}[$ BLS10]. But for generic $R$, the integrals (4.26) are non-local operators, and the deformation map $\phi\left(f_{n}\right) \mapsto \phi_{R, Q}\left(f_{n}\right)$ takes a different form. The extension of this map to bounded operators and its integral representations will be discussed in a forthcoming publication with J. Schlemmer.

We now show that the deformation $\phi(f) \mapsto \phi_{R, Q}(f)$ produces in fact new models, which are not equivalent to their undeformed counterparts. To this end, we will compute the two-particle scattering of the deformed models defined by the fields $\phi_{R, Q}$, following the Haag-Ruelle-Hepp approach Ara99, Hep65 in its form adapted to wedge-localized operators BBS01. Picking $f_{1}, g_{1} \in \mathscr{S}_{1}$, the fields $\phi_{R, Q}\left(f_{1}\right), \phi_{R,-Q}\left(g_{1}\right)$ are localized in the wedges $W_{0}+\operatorname{supp} f_{1}$ and $W_{0}^{\prime}+\operatorname{supp} g_{1}$, respectively, and create single particle states from the vacuum 3 :

$$
\phi_{R, \pm Q}\left(f_{1}\right) \Omega=\phi\left(f_{1}\right) \Omega=f_{1}^{+} \in \mathcal{H}_{1} .
$$

To define two-particle scattering states, we choose $f_{1}, g_{1}$ in such a way that supp $\tilde{f}_{1}$, supp $\tilde{g}_{1}$ are concentrated around points on the upper mass shell, and do not intersect the lower mass shell. Furthermore, we introduce the usual notations $\Gamma\left(f_{1}\right):=\left\{\left(1, \boldsymbol{p} / \varepsilon_{\boldsymbol{p}}\right): p \in \operatorname{supp} \tilde{f}_{1}\right\}$ for the velocity support of $f_{1}$, and $f_{1, t}(x):=(2 \pi)^{-d / 2} \int d p \tilde{f}(p) e^{i\left(p^{0}-\varepsilon_{\boldsymbol{p}}\right) t} e^{-i p \cdot x}$, with $p=\left(p^{0}, \boldsymbol{p}\right)$ and $\varepsilon_{\boldsymbol{p}}=\left(\boldsymbol{p}^{2}+m^{2}\right)^{1 / 2}$, for its Klein-Gordon time evolution. It is well known that for asymptotic times $t$, the support of $f_{1, t}$ is essentially contained in $t \Gamma\left(f_{1}\right)$ Hep65, that is, the restriction of $f_{1, t}$ to the complement of an open neighborhood of $t \Gamma\left(f_{1}\right)$ converges to zero in the topology of $\mathscr{S}_{1}$ as $|t| \rightarrow \infty$.

\footnotetext{
${ }^{2}$ I acknowledge helpful discussions with Sergio Yuhjtman about this question.

${ }^{3}$ In fact, these fields are temperate polarization-free generators in the sense of [BBS01].
} 
Because of the compact supports of $f_{1}, g_{1}$ in momentum space, the fields $\phi_{R, Q}\left(f_{1}\right)$ and $\phi_{R,-Q}\left(g_{1}\right)$ are not sharply localized in Minkowski space. However, for asymptotic times we have localization of $\phi_{R, Q}\left(f_{1, t}\right)$ and $\phi_{R,-Q}\left(g_{1}\right)$ in $W_{0}+t \Gamma\left(f_{1}\right)$ and $W_{0}^{\prime}+t \Gamma\left(g_{1}\right)$, respectively. If the velocity supports of $f_{1}, g_{1}$ lie in a suitable relative position to the wedge $W_{0}$, namely $\Gamma\left(f_{1}\right)-\Gamma\left(g_{1}\right) \subset W_{0}$, these regions are spacelike for $t>0$. As $t \rightarrow \infty$, we therefore find two-particle outgoing scattering states as the limits BBS01

$$
\lim _{t \rightarrow \infty} \phi_{R,-Q}\left(g_{1, t}\right) \phi_{R, Q}\left(f_{1, t}\right) \Omega=\lim _{t \rightarrow \infty} \phi_{R, Q}\left(f_{1, t}\right) \phi_{R-Q}\left(g_{1, t}\right) \Omega=: f_{1}^{+} \times_{\text {out }}^{R} g_{1}^{+} .
$$

To construct scattering states of incoming particles, the ordering of $f_{1}, g_{1}$ has to be reversed: For $t<0$, the localization regions $W_{0}+t \Gamma\left(g_{1}\right)$ and $W_{0}^{\prime}+t \Gamma\left(f_{1}\right)$ lie spacelike if $\Gamma\left(f_{1}\right)-\Gamma\left(g_{1}\right) \subset W_{0}$, and we have

$$
\lim _{t \rightarrow-\infty} \phi_{R,-Q}\left(f_{1, t}\right) \phi_{R, Q}\left(g_{1, t}\right) \Omega=\lim _{t \rightarrow-\infty} \phi_{R, Q}\left(g_{1, t}\right) \phi_{R,-Q}\left(f_{1, t}\right) \Omega=: f_{1}^{+} \times_{\mathrm{in}}^{R} g_{1}^{+} .
$$

All these limits are easy to compute in the present setting. Since the supports of $f_{1}, g_{1}$ do not intersect the lower mass shell, the annihilation parts of the fields drop out, and because the $t$-dependence of $f_{1, t}$ is trivial on the upper mass shell, one finds, $\Gamma\left(f_{1}\right)-\Gamma\left(g_{1}\right) \subset W_{0}$,

$$
\begin{aligned}
f_{1}^{+} \times_{\text {out }}^{R} g_{1}^{+} & =\lim _{t \rightarrow \infty} \phi_{R, Q}\left(f_{1, t}\right) \phi_{R,-Q}\left(g_{1, t}\right) \Omega=a_{R, Q}^{\dagger}\left(f_{1}^{+}\right) a^{\dagger}\left(g_{1}^{+}\right) \Omega, \\
f_{1}^{+} \times_{\text {in }}^{R} g_{1}^{+} & =\lim _{t \rightarrow-\infty} \phi_{R,-Q}\left(f_{1, t}\right) \phi_{R, Q}\left(g_{1, t}\right) \Omega=a_{R,-Q}^{\dagger}\left(f_{1}^{+}\right) a^{\dagger}\left(g_{1}^{+}\right) \Omega .
\end{aligned}
$$

These two-particle vectors have the explicit form

$$
\begin{aligned}
\left(f_{1}^{+} \times_{\text {out } / \text { in }}^{R} g_{1}^{+}\right)\left(p_{1}, p_{2}\right) & =\left(a_{R, \pm Q}^{\dagger}\left(f_{1}^{+}\right) g_{1}^{+}\right)_{2}\left(p_{1}, p_{2}\right) \\
& =\frac{1}{\sqrt{2}}\left(R\left( \pm p_{1} \cdot Q p_{2}\right) f_{1}^{+}\left(p_{1}\right) g_{1}^{+}\left(p_{2}\right)+R\left( \pm p_{2} \cdot Q p_{1}\right) f_{1}^{+}\left(p_{2}\right) g_{1}^{+}\left(p_{1}\right)\right) .
\end{aligned}
$$

To compute S-matrix elements, let $f_{1}, g_{1}, h_{1}, k_{1} \in \mathscr{S}_{1}$ with $\Gamma\left(f_{1}\right)-\Gamma\left(g_{1}\right) \subset W_{0}, \Gamma\left(h_{1}\right)-\Gamma\left(k_{1}\right) \subset$ $W_{0}$. Taking into account these momentum space supports yields the scalar products

$$
\left\langle f_{1}^{+} \times_{\text {out }}^{R} g_{1}^{+}, h_{1}^{+} \times_{\text {in }}^{R} k_{1}^{+}\right\rangle=\int d \mu\left(p_{1}\right) d \mu\left(p_{2}\right) R\left(-p_{1} \cdot Q p_{2}\right)^{2} \overline{\tilde{f}_{1}\left(p_{1}\right)} \overline{\tilde{g}_{1}\left(p_{2}\right)} \tilde{h}_{1}\left(p_{1}\right) \widetilde{k}_{1}\left(p_{2}\right) .
$$

This formula shows that the S-Matrix elements of the discussed model depends on the deformation. In particular, the scattering in the undeformed theory, corresponding to $R(a)=1$, and the deformed one is different, and the deformed theory is not equivalent to the undeformed one.

Equation (4.30) also clarifies the role of the function $R$ on which our deformation is based: The elastic two-particle S-Matrix kernels of the undeformed and deformed theory differ by its square $R\left(-p_{1} \cdot Q p_{2}\right)^{2}$. Since $R$ is a phase factor, the effects in collision processes are relatively small, and can only be measured in special setups such as time delay experiments. These features are similar to the properties of the S-matrices found in the warped convolution deformation GL08, BS08.

In view of the dependence of the S-matrix on $Q$, which is only invariant under the boosts preserving $W_{0}$, but not the full Lorentz group in $d>1+1$ dimensions, we also observe that the two-particle S-matrix obtained here is not fully Lorentz invariant in $d>1+1$. As a consequence, it follows that the model theory constructed here can not contain many observables localized in 
bounded spacetime regions $O$. When passing to von Neumann algebras of observables localized in $O$, one finds that at least the Reeh-Schlieder property is violated.

In $d=1+1$ dimensions, however, the identity component of the Lorentz group consists just of the one-dimensional boost group, and hence the above S-matrix is fully Lorentz invariant in this case. We will discuss the two-dimensional situation in Section 6 .

\section{Modular structure}

In this section we explain how to pass from the unbounded field operators $\phi_{R, Q}(f), f \in \underline{\mathscr{S}}$, to associated von Neumann algebras, and study their modular structure. The first step is to control commutators of bounded functions of fields.

Proposition 5.1 Let $R$ be a deformation function, and $Q$ an admissible matrix.

i.) Let $f_{1}=f_{1}^{*} \in \mathscr{S}_{1}$. Then $\phi_{R, Q}(f)$ is essentially self-adjoint.

ii.) Let $f_{1}=f_{1}^{*} \in \mathscr{S}_{1}\left(W_{0}\right)$ and $g_{1}=g_{1}^{*} \in \mathscr{S}_{1}\left(W_{0}^{\prime}\right)$. Then the self-adjoint closures $\overline{\phi_{R, Q}\left(f_{1}\right)}$ and $\overline{\phi_{R,-Q}\left(g_{1}\right)}$ commute, i.e.,

$$
\left[e^{i t \overline{\phi_{R, Q}\left(f_{1}\right)}}, e^{i s \overline{\phi_{R,-Q}\left(g_{1}\right)}}\right]=0, \quad t, s \in \mathbb{R} .
$$

Proof: i) We will first show that any $\Psi \in \mathcal{D}$ is an entire analytic vector for the field operators $\phi_{R, Q}\left(f_{1}\right), f_{1} \in \mathscr{S}_{1}$. For $\varphi \in \mathcal{H}_{1}$, the annihilation operator $a_{R, Q}(\varphi)$ can be estimated with the help of (4.23) and $|R(t)|=1$ as

$$
\begin{aligned}
\left|\left(a_{R, Q}(\varphi) \Psi_{n}\right)\left(p_{1}, \ldots, p_{n-1}\right)\right| & \leq \sqrt{n}\left|\int d \mu(q) \varphi(q) \prod_{k=1}^{n} R\left(Q q \cdot p_{k}\right) \Psi_{n}\left(q, p_{1}, \ldots, p_{n-1}\right)\right| \\
& \leq \sqrt{n} \int d \mu(q)|\varphi(q)|\left|\Psi_{n}\left(q, p_{1}, \ldots, p_{n-1}\right)\right| .
\end{aligned}
$$

By standard $L^{2}$-estimates, this implies $\left\|\left.a_{R, Q}(\varphi)\right|_{\mathcal{H}_{n}}\right\| \leq \sqrt{n}\|\varphi\|$, and taking adjoints, also $\left\|\left.a_{R, Q}^{\dagger}(\varphi)\right|_{\mathcal{H}_{n}}\right\| \leq \sqrt{n+1}\|\varphi\|$ follows. Thus we have the basic bound

$$
\left\|\left.\phi_{R, Q}\left(f_{1}\right)\right|_{\mathcal{H}_{n}}\right\| \leq \sqrt{n+1}\left(\left\|f_{1}^{+}\right\|_{\mathcal{H}_{1}}+\left\|f_{1}^{-}\right\|_{\mathcal{H}_{1}}\right) .
$$

With this bound one can easily show that any $\Psi \in \mathcal{D}$ is an entire analytic vector for $\phi_{R, Q}\left(f_{1}\right)$ (see, for example, the proof of Theorem X.41 in [RS75]). As $\mathcal{D} \subset \mathcal{H}$ is dense, application of Nelson's analytic vector theorem [RS75, Thm. X.39] shows that $\phi_{R, Q}\left(f_{1}\right), f_{1}^{*}=f_{1}$, is essentially self-adjoint. Its self-adjoint closure will be denoted $\overline{\phi_{R, Q}\left(f_{1}\right)}$.

ii) Using the bound (5.2) again, one also shows that $e^{i s \overline{\phi_{R,-Q}\left(g_{1}\right)}} \Psi, g_{1}=g_{1}^{*} \in \mathscr{S}_{1}, s \in \mathbb{R}$, $\Psi \in \mathcal{D}$, is an entire analytic vector for $\overline{\phi_{R, Q}\left(f_{1}\right)}$, as in free field theory. Hence on $\Psi \in \mathcal{D}$, the commutator (5.1) can be computed as the power series

$$
\left[e^{i t \overline{\phi_{R, Q}\left(f_{1}\right)}}, e^{i s \overline{\phi_{R,-Q}\left(g_{1}\right)}}\right] \Psi=\sum_{n, n^{\prime}=0}^{\infty} \frac{i^{n+n^{\prime}} t^{n} s^{n^{\prime}}}{n ! n^{\prime} !}\left[\phi_{R, Q}\left(f_{1}\right)^{n}, \phi_{R,-Q}\left(g_{1}\right)^{n^{\prime}}\right] \Psi .
$$

As $\phi_{R, Q}\left(f_{1}\right)$ and $\phi_{R,-Q}\left(g_{1}\right)$ commute on $\phi(\underline{\mathscr{S}}) \Omega$ (Proposition 4.1]证), the proof is finished. 
We now introduce the von Neumann algebras generated by the self-adjoint field operators,

$$
\begin{aligned}
& \mathcal{M}_{R, Q}:=\left\{e^{i \overline{\phi_{R, Q}\left(f_{1}\right)}}: f_{1}=f_{1}^{*} \in \mathscr{S}_{1}\left(W_{0}\right)\right\}^{\prime \prime}, \\
& \widehat{\mathcal{M}}_{R, Q}:=\left\{e^{i \overline{\phi_{R,-Q}\left(g_{1}\right)}}: g_{1}=g_{1}^{*} \in \mathscr{S}_{1}\left(W_{0}^{\prime}\right)\right\}^{\prime \prime} \text {. }
\end{aligned}
$$

In view of Proposition 5.1 ii), these algebras commute, $\widehat{\mathcal{M}}_{R, Q} \subset \mathcal{M}_{R, Q}{ }^{\prime}$. By standard arguments making use of the Reeh-Schlieder property established in Proposition 4.1 0 ), it also follows that the vacuum vector is cyclic for $\mathcal{M}_{R, Q}$ and $\widehat{\mathcal{M}}_{R, Q}$. As these algebras commute, $\Omega$ is separating as well. Thus Tomita Takesaki modular theory applies to the pair $\left(\mathcal{M}_{R, Q}, \Omega\right)$, and provides us with modular unitaries $\Delta_{R, Q}^{i t}$ and a modular involution $J_{R, Q}$. In the following theorem, we show that these data are stable under the deformation, i.e. do not depend on $R$ and $Q$ within the specified limitations. For the special case $R(a)=e^{i a}$, this fact was already shown in [BLS10].

Theorem 5.2 Let $R$ be a deformation function and $Q$ an admissible matrix.

i.) The modular data $J_{R, Q}, \Delta_{R, Q}$ of $\mathcal{M}_{R, Q}, \Omega$ are independent of $R$ and $Q$.

ii.) The Bisognano-Wichmann property holds,

$$
\Delta_{R, Q}^{i t}=U\left(0, \Lambda_{1}(2 \pi t)\right), \quad J_{R, Q}=U(0, j),
$$

with $\Lambda_{1}(t):\left(x^{0}, \ldots, x^{d-1}\right) \mapsto\left(\cosh (t) x^{0}+\sinh (t) x^{1}, \sinh (t) x^{0}+\cosh (t) x^{1}, x^{2}, \ldots, x^{d-1}\right)$ denoting the boosts in $x^{1}$-direction.

iii.) $\widehat{\mathcal{M}}_{R, Q}=\mathcal{M}_{R, Q}{ }^{\prime}$.

Proof: We first show that given $f \in \underline{\mathscr{L}}\left(W_{0}\right)$, the closed operator $F:=\overline{\phi_{R, Q}(f)}$ is affiliated with $\mathcal{M}_{R, Q}$. To this end, let $\Psi \in \operatorname{dom} F, \Psi_{0} \in \mathcal{D}$, and consider a real test function $g_{1}^{\prime} \in \mathscr{S}_{1}\left(W_{0}^{\prime}\right)$. As $F^{*}$ changes the particle number only be a finite amount, both $\Psi_{0}$ and $F^{*} \Psi_{0}$ are entire analytic vectors for $G^{\prime}:=\overline{\phi_{R,-Q}\left(g_{1}^{\prime}\right)}$. Taking also into account that $F^{*}$ and $\left(G^{\prime}\right)^{p}$ commute on $\mathcal{D}$ for any $p \in \mathbb{N}_{0}$ (Proposition 4.1]iv), we find

$$
\begin{aligned}
\left\langle\Psi_{0}, e^{i G^{\prime}} F \Psi\right\rangle & =\left\langle e^{-i G^{\prime}} \Psi_{0}, F \Psi\right\rangle=\sum_{p=0}^{\infty} \frac{(-i)^{p}}{p !}\left\langle F^{*}\left(G^{\prime}\right)^{p} \Psi_{0}, \Psi\right\rangle=\sum_{p=0}^{\infty} \frac{(-i)^{p}}{p !}\left\langle\left(G^{\prime}\right)^{p} F^{*} \Psi_{0}, \Psi\right\rangle \\
& =\left\langle e^{-i G^{\prime}} F^{*} \Psi_{0}, \Psi\right\rangle=\left\langle\Psi_{0}, F e^{i G^{\prime}} \Psi\right\rangle .
\end{aligned}
$$

As $\mathcal{D} \subset \mathcal{H}$ is dense, this implies $e^{i G^{\prime}} F \Psi=F e^{i G^{\prime}} \Psi$. Clearly, this identity then also holds when $e^{i G^{\prime}}$ is replaced by any operator in the ${ }^{*}$-algebra $\mathcal{A}$ generated (algebraically) by the $e^{i \overline{\phi_{R,-Q}\left(g_{1}^{\prime}\right)}}, g_{1}^{\prime} \in \mathscr{S}_{1}\left(W_{0}^{\prime}\right)$ real. But any $A^{\prime} \in \mathcal{M}_{R, Q}^{\prime}$ is a weak limit of a sequence $A_{n}^{\prime}$ in $\mathcal{A}$, and $A_{n}^{\prime} F \Psi=F A_{n}^{\prime} \Psi$ is stable under weak limits. Thus we arrive at $A^{\prime} F \Psi=F A^{\prime} \Psi$ for all $\Psi \in \operatorname{dom} F$, i.e., $F$ is affiliated with $\mathcal{M}_{R, Q}$.

Proceeding to the polar decomposition $F=V|F|$ and the spectral projections $E_{n}$ of $|F|$ onto spectrum in the interval $[0, n]$, we have $V, E_{n}|F| \in \mathcal{M}_{R, Q}$ for all $n \in \mathbb{N}$. Now let $S_{R, Q}$ denote the Tomita operator of $\left(\mathcal{M}_{R, Q}, \Omega\right)$. As $S_{R, Q} V E_{n}|F| \Omega=|F| E_{n} V^{*} \Omega$, the strong convergence $E_{n} \rightarrow 1$ as $n \rightarrow \infty$ and the closedness of $S_{R, Q}$ imply that $F \Omega$ lies in the domain of $S_{R, Q}$, and $S_{R, Q} F \Omega=F^{*} \Omega$.

As all these considerations apply in particular to the special case $R=1$, we have now gathered sufficient information for establishing $i$ ). Let $S$ denote the Tomita operator of the 
undeformed algebra $\mathcal{M}:=\mathcal{M}_{1, Q}$ w.r.t. $\Omega$, and let $f \in \underline{\mathscr{S}}\left(W_{0}\right)$ as above. Making use of (4.6), we find

$$
S_{R, Q} \phi(f) \Omega=S_{R, Q} \phi_{R, Q}(f) \Omega=\phi_{R, Q}(f)^{*} \Omega=\phi_{R, Q}\left(f^{*}\right) \Omega=\phi\left(f^{*}\right) \Omega=\phi(f)^{*} \Omega=S \phi(f) \Omega,
$$

i.e., $S_{R, Q}$ and $S$ coincide on the subspace $\phi\left(\underline{\mathscr{S}}\left(W_{0}\right)\right) \Omega$. But this domain is a core for $S=J \Delta^{1 / 2}$ because it is dense and the modular group $\Delta^{i t}$ acts as the Lorentz boosts $\Lambda_{1}(2 \pi t)$ which leave $W_{0}$ invariant BW75]. As $S$ and $S_{R, Q}$ are closed operators, this shows that $S_{R, Q}$ is an extension of $S$, i.e., $S_{R, Q} \supset S$.

We now consider the commutants $\mathcal{M}_{R, Q}^{\prime}, \mathcal{M}^{\prime}$. By modular theory, their Tomita operators w.r.t. $\Omega$ are the adjoints $S_{R, Q}^{*}, S^{*}$. In complete analogy to above, one can show that for $f^{\prime} \in \underline{\mathscr{S}}\left(W_{0}^{\prime}\right)$, the operator $\overline{\phi_{R,-Q}\left(f^{\prime}\right)}$ is affiliated with $\mathcal{M}_{R, Q}^{\prime}$, and

$$
S_{R, Q}^{*} \phi\left(f^{\prime}\right) \Omega=S_{R, Q}^{*} \phi_{R,-Q}\left(f^{\prime}\right) \Omega=\phi_{R,-Q}\left(f^{\prime}\right)^{*} \Omega=\phi\left(f^{\prime}\right)^{*} \Omega=S^{*} \phi\left(f^{\prime}\right) \Omega .
$$

Since $\phi\left(W_{0}^{\prime}\right) \Omega$ is a core for $S^{*}$, this shows $S_{R, Q}^{*} \supset S^{*}$, or, equivalently, $S_{R, Q}=S_{R, Q}^{* *} \subset S^{* *}=S$. Together with the previously established extension $S_{R, Q} \supset S$, this yields $S_{R, Q}=S$. The identities $\Delta_{R, Q}=\Delta$ and $J_{R, Q}=J$ then follow from the uniqueness of the polar decomposition $S_{R, Q}=J_{R, Q} \Delta_{R, Q}^{1 / 2}$.

As the Bisognano-Wichmann property (5.3) is known to hold for the free field theory [BW75, ii) follows immediately from $i$ ). The transformation law (4.8) of the field implies $U(0, j) \mathcal{M}_{R, Q} U(0, j)=\widehat{\mathcal{M}}_{R, Q}$ by extension from analytic vectors. By Tomita's theorem, this yields

$$
\widehat{\mathcal{M}}_{R, Q}=U(0, j) \mathcal{M}_{R, Q} U(0, j)=J_{R, Q} \mathcal{M}_{R, Q} J_{R, Q}=\mathcal{M}_{R, Q}{ }^{\prime},
$$

which proves iii).

Von Neumann algebras with modular data identical to the geometric ones found in free field theory have been studied as a possible tool in the construction of quantum field theories before [Wol92, LMW00]. It is therefore interesting to note that the deformation construction presented here establishes a new infinite family of solutions to this inverse problem in modular theory.

For a formulation of our models in the framework of algebraic quantum field theory, we now consider the von Neumann algebras

$$
\mathcal{A}_{R}(W):=U(x, \Lambda) \mathcal{M}_{R, Q} U(x, \Lambda)^{-1},
$$

where $W$ is a wedge and $(x, \Lambda) \in \mathcal{P}_{+}$is any Poincaré transformation satisfying $\Lambda W_{0}+x=W$. (We have suppressed the dependence of the left hand side on $Q$ here because $Q$ is transformed by $\Lambda$.) The transformation behaviour of the field $\phi_{R, Q}(f)$ implies that (5.4) is well-defined, i.e. independent of the choice of $(x, \Lambda)$. Furthermore, we have, $W, \tilde{W} \in \mathcal{W}$,

$$
\begin{aligned}
\mathcal{A}_{R}(W) & \subset \mathcal{A}_{R}(\tilde{W}) \quad \text { for } W \subset \tilde{W}, \\
\mathcal{A}_{R}(W) & =\mathcal{A}_{R}\left(W^{\prime}\right)^{\prime} \\
U(x, \Lambda) \mathcal{A}_{R}(W) U(x, \Lambda)^{-1} & =\mathcal{A}_{R}(\Lambda W+x),
\end{aligned}
$$

where in the last line, $(x, \Lambda) \in \mathcal{P}_{+}$is arbitrary. In view of the unitarity of $U$, it is also clear that $\Omega$ is cyclic and separating for each $\mathcal{A}_{R}(W), W \in \mathcal{W}$. We summarize these findings in the following proposition. 
Proposition 5.3 Let $R$ be a deformation function and $Q$ an admissible matrix. Then the map $\mathcal{A}_{R}: \mathcal{W} \ni W \longmapsto \mathcal{A}_{R}(W) \subset \mathcal{B}(\mathcal{H})$ (5.4) is an isotonous, Haag-dual net of von Neumann algebras which transforms covariantly under the adjoint action of $U$, and the vacuum vector $\Omega$ is cyclic and separating for each $\mathcal{A}_{R}(W), W \in \mathcal{W}$.

As is well known, it is possible to extend a net with the above properties to arbitrary regions in Minkowski space by taking suitable intersections of the algebras $\mathcal{A}_{R}(W)$. This extension always preserves isotony, locality, and covariance. However, the algebras associated with bounded regions might be small or even trivial.

In the case at hand, the results found in the computation of the two-particle scattering states in the previous section imply that $\Omega$ is not cyclic for algebras associated with bounded regions in dimension $d>1+1$. In two space-time dimensions, the situation is however different. This case will be discussed in the next section.

\section{Integrable models as deformations of free field theories}

Up to this point, the dimension $d \geq 1+1$ of spacetime did not play any role in our constructions. Now we will consider the special case $d=1+1$ of a two-dimensional Minkowski space. The matrix $Q$ appearing in the deformation two point function then has the form (3.28)

$$
Q=\lambda\left(\begin{array}{ll}
0 & 1 \\
1 & 0
\end{array}\right), \quad \lambda \in \mathbb{R} .
$$

In two dimensions, it is convenient to parametrize the upper mass shell of mass $m>0$ by the rapidity $\theta \in \mathbb{R}$ according to $p(\theta):=m(\cosh \theta, \sinh \theta)$. Inserting this parametrization into the deformation two point function (3.26) yields

$$
\rho_{2}\left(p\left(\theta_{1}\right), p\left(\theta_{2}\right)\right)=R\left(-p\left(\theta_{1}\right) \cdot Q p\left(\theta_{2}\right)\right)=R\left(\lambda m^{2} \sinh \left(\theta_{1}-\theta_{2}\right)\right), \quad \theta_{1}, \theta_{2} \in \mathbb{R},
$$

and we denote the square of this function by

$$
S_{\lambda}: \mathbb{R} \rightarrow \mathbb{C}, \quad S_{\lambda}(\theta):=R\left(\lambda m^{2} \sinh \theta\right)^{2} .
$$

As mentioned earlier, $R$ is analytic on the upper half plane because of the half-sided support of its Fourier transform. As the hyperbolic sine is an entire function mapping the $\operatorname{strip} S(0, \pi):=$ $\{\zeta \in \mathbb{C}: 0<\operatorname{Im} \zeta<\pi\}$ onto the upper half plane, this implies that $S_{\lambda}, \lambda \geq 0$, extends to an analytic function on $S(0, \pi)$, with distributional boundary values at $\mathbb{R}$ and $\mathbb{R}+i \pi$. From the properties (3.22) of $R$ and sinh, it is obvious that

$$
\overline{S_{\lambda}(\theta)}=S_{\lambda}(\theta)^{-1}=S_{\lambda}(-\theta)=S_{\lambda}(\theta+i \pi), \quad \lambda, \theta \in \mathbb{R} .
$$

These relations are well known from the analysis of completely integrable quantum field theories with factorizing S-matrices on two-dimensional Minkowski space [AAR91, where they express the unitarity, hermitian analyticity, and crossing symmetry [BFK06] of a two-particle S-matrix of such a model. Here these properties show up as a consequence of our deformation construction.

Not only the typical relations of a factorizing S-matrix appear here, but also the characteristic algebraic structure known as the Zamolodchikov-Faddeev algebra [ZZ79, Fad84]: For the 
rapidity space creation/annihilation operators $z_{\lambda}(\theta):=a_{R, Q}(p(\theta)), z_{\lambda}^{\dagger}(\theta):=a_{R, Q}^{\dagger}(p(\theta))$, the relations (4.24) (with both $Q$ and $Q^{\prime}$ replaced by (6.1)) read

$$
\begin{aligned}
& z_{\lambda}\left(\theta_{1}\right) z_{\lambda}\left(\theta_{2}\right)=S_{\lambda}\left(\theta_{1}-\theta_{2}\right) z_{\lambda}\left(\theta_{2}\right) z_{\lambda}\left(\theta_{1}\right) \\
& z_{\lambda}^{\dagger}\left(\theta_{1}\right) z_{\lambda}^{\dagger}\left(\theta_{2}\right)=S_{\lambda}\left(\theta_{1}-\theta_{2}\right) z_{\lambda}^{\dagger}\left(\theta_{2}\right) z_{\lambda}^{\dagger}\left(\theta_{1}\right) \\
& z_{\lambda}\left(\theta_{1}\right) z_{\lambda}^{\dagger}\left(\theta_{2}\right)=S_{\lambda}\left(\theta_{2}-\theta_{1}\right) z_{\lambda}^{\dagger}\left(\theta_{2}\right) z_{\lambda}\left(\theta_{1}\right)+\delta\left(\theta_{1}-\theta_{2}\right) \cdot 1 .
\end{aligned}
$$

This is precisely the Zamolodchikov-Faddeev algebra. In the context of factorizing S-matrices, it is mostly used as an auxiliary structure to organize $n$-particle scattering states (see, for example, CA01]). However, it is also possible to take it as a starting point for the construction of model theories.

This latter point of view has been taken by Schroer, who suggested to use the fields $\phi_{\lambda}(x):=\int d \theta\left(e^{i p(\theta) \cdot x} z_{\lambda}^{\dagger}(\theta)+e^{-i p(\theta) \cdot x} z_{\lambda}(\theta)\right)$ as wedge-local polarization-free generators for constructing quantum field theories [Sch97]. Although this construction was originally formulated independently of deformation ideas, the same fields also appear in the present setting, and coincide with the deformed fields $\phi_{R, Q}$ from the previous section. In the two-dimensional context, their properties as listed in Proposition 4.1 were known already in case the scattering function $S$ satisfies (6.4) and is analytic and bounded on the strip $S(0, \pi)$ Lec03.

Full-fledged quantum field theories based on these deformed fields have been constructed in the framework of algebraic quantum field theory [Haa96]: After passing from the wedge-local fields to corresponding nets of von Neumann algebras, operator-algebraic techniques become available for the analysis of the local observable content of these models BL04. We recall from [Lec06, Lec08] that if $S$ is regular in the sense that it has a bounded analytic extension to the strip $\{\zeta \in \mathbb{C}:-\varepsilon<\operatorname{Im} \zeta<\pi+\varepsilon\}$ for some $\varepsilon>0$, then the quantum field theory generated by $\phi_{\lambda}$ contains observables localized in double cones, at least for the radius of the double cone above some minimal size. In fact, there exist so many such local observables that they generate dense subspaces from the vacuum, as it is typical in quantum field theory (ReehSchlieder property). Also all other standard properties of quantum field theory are satisfied by these models, and the factorizing S-matrix with scattering function $S$ can be recovered from their $n$-particle collision states [Lec08. We note this relation between multiplicative deformations and integrable models as the following theorem.

Theorem 6.1 On two-dimensional Minkowski space, every integrable quantum field theory with scattering function $S$ of the form (6.3) arises from a free field theory by a (multiplicative) deformation. If $S$ is regular, then the deformed theory is local in the sense that the vacuum is cyclic for all observable algebras associated with double cones above a minimal size [Lec08, Thm. 5.6].

Although the structure of integrable quantum field theories is quite simple, the important message for the deformation technique presented here is that this method is capable of deforming covariant local free quantum field theories to covariant local interacting quantum field theories. For the deformed models to contain sufficiently many local observables, we only have to select the deformation function $R$ in such a way that $S(6.3)$ is regular. For example, this is the case for the finite Blaschke products

$$
R(a)=\prod_{k=1}^{N} \frac{z_{k}-a}{z_{k}+a},
$$

where the zeros $z_{1}, \ldots, z_{N}$ lie in the upper half plane and occur in pairs $z_{k},-\overline{z_{k}}(\underline{3.24})$. 


\section{Conclusions}

In this paper we have established a family of deformations of quantum field theories, leading to new models with non-trivial interaction in any number of space-time dimensions $d$. This result supports the general deformation approach, and shows that it is possible to use deformation methods for obtaining interacting local field theories from models without interaction. As interacting quantum field theories in physical spacetime must necessarily involve particle production processes [Aks65, and particle production was ruled out here because of the relatively simple form of the multiplicative deformations, the obtained models are not yet physically realistic. In two space-time dimensions, they have the structure of integrable models, and there are indications that the family of integrable models which can be realized in this manner is actually much larget 4 . For models on higher-dimensional Minkowski space, however, one needs to allow for particle production processes already on the level of the deformation maps, and replace the multiplicative deformations by more general integral operators (3.4). Apart from these modifications, it seems to be possible to use the same approach as presented here to realize also interactions with momentum transfer and particle production by deformation methods.

From a structural point of view, it is desirable to uncouple the deformations from the specific form of the Borchers-Uhlmann tensor algebra. This has been achieved in the case of the warped convolutions [BS08, which are formulated in such a way that they are applicable to any vacuum quantum field theory [BLS10]. Such an operator-algebraic reformulation of the deformations studied here is currently under investigation.

Regarding the operator-algebraic structure, we have shown that the modular data of the von Neumann algebras associated with wedge-local deformed quantum fields represented in compatible states are identical to those in the undeformed theory. This is essentially a consequence of the compatibility of the deformations with the ${ }^{*}$-involution and unit element of $\mathscr{L}$, and can therefore be expected to be a generic feature of deformations of quantum field theories. This feature connects our deformation approach to another approach to the construction of quantum field theories, based on the inverse problem in modular theory [LMW00]. Furthermore, also the root of the S-matrix plays a role in both, our present setting, where it appears in the deformation two-point function, and in the context of inverse problems in modular theory, where it is used to identify modular conjugations [Wol92]. These interesting connections require further investigation, which will be presented elsewhere.

\section{Acknowledgements}

I enjoyed helpful discussions in the Vienna deformation group, in particular with Sabina Alazzawi, Jan Schlemmer, Jakob Yngvason, and Sergio Yuhjtman. Many thanks go also to Stefan Waldmann for informing me about the rigidity of tensor algebras.

\section{References}

[AAR91] E. Abdalla, C. Abdalla, and K.D. Rothe. Non-perturbative methods in 2-dimensional quantum field theory. World Scientific, 1991.

[Åks65] S. Åks. Proof that scattering implies production in quantum field theory. J. Math. Phys. 6 (1965) 516-532.

\footnotetext{
${ }^{4}$ S. Alazzawi, C. Schützenhofer, work in progress.
} 
[Ara99] H. Araki. Mathematical Theory of Quantum Fields. Int. Series of Monographs on Physics. Oxford University Press, Oxford, 1999.

[BBS01] H.-J. Borchers, D. Buchholz, and B. Schroer. Polarization-free generators and the S-matrix. Commun. Math. Phys. 219 (2001) 125-140, [open access].

[BFK06] H. M. Babujian, Angela Foerster, and Michael Karowski. The Form Factor Program: a Review and New Results - the Nested SU(N) Off-Shell Bethe Ansatz. SIGMA 2 (2006) 082, [open access].

[BGL02] R. Brunetti, D. Guido, and R. Longo. Modular localization and Wigner particles. Rev. Math. Phys. 14 (2002) 759-786, [open access].

[BL04] D. Buchholz and G. Lechner. Modular nuclearity and localization. Annales Henri Poincaré 5 (2004) 1065-1080, [open access].

[BLS10] D. Buchholz, G. Lechner, and S. J. Summers. Warped Convolutions, Rieffel Deformations and the Construction of Quantum Field Theories. Commun. Math. Phys. (2010), [open access].

[Bor62] H.-J. Borchers. On Structure of the Algebra of Field Operators. Nuovo Cimento 24 (1962)

[Bor92] H.-J. Borchers. The CPT theorem in two-dimensional theories of local observables. Commun. Math. Phys. 143 (1992) 315-332.

[BS07] D. Buchholz and S. J. Summers. String- and brane-localized fields in a strongly nonlocal model. J. Phys. A40 (2007) 2147-2163, [open access].

[BS08] D. Buchholz and S. J. Summers. Warped Convolutions: A Novel Tool in the Construction of Quantum Field Theories. In E. Seiler and K. Sibold, editors, Quantum Field Theory and Beyond: Essays in Honor of Wolfhart Zimmermann, pages 107121. World Scientific, 2008. [open access].

[Buc90] D. Buchholz. On quantum fields that generate local algebras. J. Math. Phys. 31 (1990) 1839-1846.

[BW75] J. J Bisognano and E. H. Wichmann. On the Duality Condition for a Hermitian Scalar Field. J. Math. Phys. 16 (1975) 985-1007.

[BW92] H. Baumgärtel and M. Wollenberg. Causal Nets of Operator Algebras. Akademie Verlag, 1992.

[BY90] H.-J. Borchers and J. Yngvason. Positivity of Wightman functionals and the existence of local nets. Commun. Math. Phys. 127 (1990) 607, [open access].

[BZ63] H.-J. Borchers and W. Zimmermann. On the Self-Adjointness of Field Operators. Nuovo Cimento 31 (1963) 1047-1059.

[CA01] O. Castro-Alvaredo. Bootstrap Methods in 1+1-Dimensional Quantum Field Theories: the Homogeneous Sine-Gordon Models. PhD thesis, Santiago de Compostela, 2001. [open access]. 
[DLM11] C. Dappiaggi, G. Lechner, and E. Morfa-Morales. Deformations of quantum field theories on spacetimes with Killing fields. Commun. Math. Phys. (2011), [open access].

[DSW86] W. Driessler, S. J. Summers, and E. H. Wichmann. On the Connection between Quantum Fields and von Neumann Algebras of Local Operators. Commun. Math. Phys. 105 (1986) 49-84.

[DT10] W. Dybalski and Y. Tanimoto. Asymptotic completeness in a class of massless relativistic quantum field theories Preprint (June, 2010), [open access].

[Fad84] L. D. Faddeev. Quantum completely integrable models in field theory, volume 1 of Mathematical Physics Reviews, pages 107-155. 1984. In Novikov, S.p. ( Ed.): Mathematical Physics Reviews, Vol. 1, 107-155.

[Ger64] M. Gerstenhaber. On the deformation of rings and algebras. Ann. Math. 79 (1964) 59-103, [open access].

[GL07] H. Grosse and G. Lechner. Wedge-Local Quantum Fields and Noncommutative Minkowski Space. JHEP 11 (2007) 012, [open access].

[GL08] H. Grosse and G. Lechner. Noncommutative Deformations of Wightman Quantum Field Theories. JHEP 09 (2008) 131, [open access].

[Haa96] R. Haag. Local Quantum Physics - Fields, Particles, Algebras. Springer, 2 edition, 1996.

[Hep65] K. Hepp. On the connection between Wightman and LSZ quantum field theory. Commun. Math. Phys. 1 (1965) 95-111.

[Jos65] R. Jost. The General Theory of Quantized Fields. 1965.

[Lec03] G. Lechner. Polarization-free quantum fields and interaction. Lett. Math. Phys. 64 (2003) 137-154, [open access].

[Lec05] G. Lechner. On the existence of local observables in theories with a factorizing S-matrix. J. Phys. A38 (2005) 3045-3056, [open access]

[Lec06] G. Lechner. On the construction of quantum field theories with factorizing S-matrices. $\mathrm{PhD}$ thesis, University of Göttingen, 2006. [open access].

[Lec08] G. Lechner. Construction of Quantum Field Theories with Factorizing S-Matrices. Commun. Math. Phys. 277 (2008) 821-860, [open access].

[LMW00] M. Leitz-Martini and M. Wollenberg. Notes on Modular Conjugations of von Neumann Factors. Z. Anal. Anw. (2000) no. 19, 13-22, [open access].

[LR04] R. Longo and K.-H. Rehren. Local fields in boundary conformal QFT. Rev. Math. Phys. 16 (2004) 909, [open access].

[LW10] R. Longo and E. Witten. An Algebraic Construction of Boundary Quantum Field Theory Preprint (April, 2010), [open access].

[MSY06] J. Mund, B. Schroer, and J. Yngvason. String-localized quantum fields and modular localization. Commun. Math. Phys. 268 (2006) 621-672, [open access]. 
[Mun10] J. Mund. An Algebraic Jost-Schroer Theorem for Massive Theories Preprint (December, 2010), [open access].

[Rie92] M. A. Rieffel. Deformation Quantization for Actions of $R^{d}$, volume 106 of Memoirs of the Amerian Mathematical Society. American Mathematical Society, Providence, Rhode Island, 1992.

[Rie93] M. A. Rieffel. Compact quantum groups associated with toral subgroups. Cont. Math. 145 (1993), [open access].

[RS75] M. Reed and B. Simon. Methods of Modern Mathematical Physics II - Fourier Analysis. Academic Press, 1975.

[Sch97] B. Schroer. Modular localization and the bootstrap-formfactor program. Nucl. Phys. B499 (1997) 547-568, [open access].

[SW64] R. F. Streater and A. Wightman. PCT, Spin and Statistics, and All That. BenjaminCummings, Reading, MA, 1964.

[SW00] B. Schroer and H. W. Wiesbrock. Modular constructions of quantum field theories with interactions. Rev. Math. Phys. 12 (2000) 301-326, [open access].

[Tre67] F. Treves. Topological vector spaces, distributions, and kernels. Academic Press, 1967.

[TW97] L. J. Thomas and E. H. Wichmann. On the causal structure of Minkowski space-time. J. Math. Phys. 38 (1997) 5044-5086.

[Uhl62] A. Uhlmann. Über die Definition der Quantenfelder nach Wightman und Haag. Wissenschaftliche Zeitschrift der Karl-Marx-Universität Leipzig 2 (1962) 213-217.

[Wol92] M. Wollenberg. Notes on Pertubations of Causal Nets of Operator Algebras. SFB 288 Preprint, N2. 36 (1992) unpublished.

[Yng81] J. Yngvason. Translationally invariant states and the spectrum ideal in the algebra of test functions for quantum fields. Commun. Math. Phys. 81 (1981) 401, [open access].

[Yng84] J. Yngvason. On the Locality Ideal in the Algebra of Test Functions for Quantum Fields. Publ. RIMS, Kyoto University (1984) no. 20, 1063-1081.

[ZZ79] A. B. Zamolodchikov and A. B. Zamolodchikov. Factorized S-matrices in two dimensions as the exact solutions of certain relativistic quantum field models. Annals Phys. 120 (1979) 253-291. 